\title{
Crystal structure and pathophysiological role of the pneumococcal nucleoside-binding protein PnrA
}

Mohammed R. Abdullah ${ }^{1,4 \S}$, María T. Batuecas ${ }^{2 \S}$, Franziska Jennert ${ }^{1,5 \S}$, Franziska Voß ${ }^{1}$, Philipp Westhoff ${ }^{3,6}$, Thomas P. Kohler ${ }^{1}$, Rafael Molina ${ }^{2}$, Stephanie Hirschmann ${ }^{1}$, Michael Lalk ${ }^{3}$, Juan A. Hermoso ${ }^{2 \#}$ and Sven Hammerschmidt ${ }^{1 \#}$

${ }^{1}$ Department of Molecular Genetics and Infection Biology, Interfaculty Institute for Genetics and Functional Genomics, Center for Functional Genomics of Microbes, University of Greifswald, D17487 Greifswald, Germany

2Department of Crystallography and Structural Biology, Institute of Physical Chemistry "Rocasolano", CSIC, 28006 Madrid, Spain.

${ }^{3}$ Cellular Metabolism / Metabolomics, Institute of Biochemistry, University of Greifswald, D-17487 Greifswald, Germany

${ }^{4}$ present address: Institut für Klinische Chemie und Laboratoriumsmedizin, Universitätsmedizin Greifswald, Germany

${ }^{5}$ present address: Institute for Microbiology, University of Veterinary Medicine Hannover, Hannover, Germany, Germany

${ }^{6}$ present address: Biochemie der Pflanzen, Heinrich-Heine-Universität Düsseldorf, Germany

Running title: Structure-function analysis of PnrA nucleoside transporter

\#Address correspondence to

Prof. Dr. Sven Hammerschmidt, Phone: +49-3834-4205700;

E-mail: sven.hammerschmidt@uni-greifswald.de

Prof. Dr. Juan A. Hermoso, Phone: +34- 917459538; E-mail: xjuan@iqfr.csic.es

§Footnote: these authors contributed equally to this work. Author order was determined alphabetically.

Abstract word count: 250

Main text word count: 7232 (5044 exclusive Methods) 


\section{Abstract}

Nucleotides are important for RNA and DNA synthesis and, despite a de novo synthesis by bacteria, uptake systems are crucial. Streptococcus pneumoniae, a facultative human pathogen, produces a surface-exposed nucleoside-binding protein, PnrA, as part of an $A B C$ transporter system. Here we demonstrate the binding affinity of PnrA to nucleosides adenosine, guanosine, cytidine, thymidine and uridine by microscale thermophoresis and indicate the consumption of adenosine and guanosine by ${ }^{1} \mathrm{H}-\mathrm{NMR}$ spectroscopy. In a series of five crystal structures we revealed the PnrA structure and provide insights into how PnrA can bind purine and pyrimidine ribonucleosides but with preference for purine ribonucleosides. Crystal structures of PnrA:nucleoside complexes unveil a clear pattern of interactions in which both the $\mathrm{N}$ - and C-domains of PnrA contribute. The ribose moiety is strongly recognized through a conserved network of $\mathrm{H}$ bond interactions, while plasticity in loop $27-36$ is essential to bind purine- or pyrimidinebased nucleosides.

Further, we deciphered the role of PnrA in pneumococcal fitness in infection experiments. Phagocytosis experiments did not show a clear difference in phagocytosis between PnrA-deficient and wild-type pneumococci. In the acute pneumonia infection model the deficiency of PnrA attenuated moderately virulence of the mutant, which is indicated by a delay in the development of severe lung infections. Importantly, we confirmed the loss of fitness in co-infections, where the wild-type out-competed the pnrA-mutant. In conclusion, we present the PnrA structure in complex with individual nucleosides and show that the consumption of adenosine and guanosine under infection conditions is required for virulence. 


\section{Introduction}

Nucleotides are essential for bacterial fitness as they are important for RNA and DNA synthesis, can serve as energy-donors for the cell, can be part of coenzymes, and are important for the activation of precursors. Besides, regulation of cellular processes such as metabolism and gene expression are influenced by nucleotides [1]. However, many lactic acid bacteria are auxotrophic for purines or pyrimidines and subsequently, uptake of nucleosides or nucleobases is critical. Nucleotides need to be taken up by cells in their dephosphorylated form, the nucleosides or nucleobases [1].

Nucleoside transporters were first described for Escherichia coli in 1976: NupG and NupC $[2,3]$. These transporters function by symport of protons. A variety of functionrelated nucleoside transporters were described in other bacteria like NupC and NupG of Bacillus subtilis $[4,5]$. However, there are no homologs known in the Gram-positive human pathobiont Streptococcus pneumoniae (the pneumococcus). While pneumococci colonize, as part of the microbiota, the upper respiratory tract as harmless commensals, they can convert into harmful pathogens causing severe lung infections and invasive infections such as septicemia and meningitis [6-8]. Pneumococcal disease caused an estimated 826,000 deaths in children under 5 years of age worldwide, accounting for $11 \%$ of all deaths in children under 5 years [9]. In addition to the expression of virulence factors, a prerequisite for pneumococcal disease sequelae is the ability of the pneumococcus to adapt to its host compartments and maintain physiological fitness. Pneumococci are versatile bacteria and the high number of phospho-transferase systems (PTS) and antigen-binding cassette (ABC)-transporters produced is a hallmark of these pathobionts [10-12]. The PTS and ABC-transporters highlight the need for 
nutrient uptake systems because pneumococci are auxotrophic e.g. for some amino acids and lack a de novo synthesis. The variety of uptakes systems ensures the maintenance of bacterial fitness under various physiological conditions in the human host [11-14].

The existence of $A B C$ transporters as a novel type of nucleoside transporter was postulated in 2006 after the crystal structure of TmpC (later renamed PnrA) of Treponema pallidum was solved and binding studies were conducted [15]. Subsequently more TmpC-like proteins were found by in silico analyses and consequently, more potential nucleoside $A B C$ transporters: BpmA-NupABC of Lactococcus lactis, NupNOPQ of B. subtilis and RnsBACD of Streptococcus mutans [5, $16,17]$.

The pneumococcal ABC transporter PnrABCD, identified earlier as a carbohydrate uptake $A B C$ transporter (CUT) $2[18,19]$, also belongs to this novel group of nucleoside transporters. CUT2 transporters are special because they contain an ATPase and two permeases, whereas the permeases consist in most cases of a homodimer encoded by just one gene. PnrABCD is suggested to be the only CUT2 transporter in the pneumococcal genome [18]. Carbohydrate transporters generally belong to the variable genome [18]. However, based on in silico studies Webb and Hosie postulated that the S. mutans PnrABCD-homologue RnsBACD is part of the core genome, since it was found in all 56 clinical isolates tested [17]. Consistently, the in silico analyses indicated that the genes encoding PnrABCD are part of the pneumococcal core genome (Figure S1). PnrA is encoded e.g. in S. pneumoniae TIGR4 by sp_0845 and in D39 by spd_0739. Because of its potential function as substrate binding protein of a nucleoside 
ABC-transporter it was designated pneumococcal nucleoside receptor A, PnrA [20]. PnrA is a highly conserved surface-exposed lipoprotein and surfacome analysis as well as flow cytometry indicated that PnrA is one of the most abundant surface-exposed pneumococcal lipoproteins [21, 22]. Interestingly, PnrA is still abundant on the pneumococcal surface in a mutant lacking the prolipoprotein diacylglyceryl transferase Lgt, which is essential for lipidation and anchoring of lipoproteins [11, 22]. Components of $A B C$ transporters are used in immunization studies and discussed as potential vaccine candidates [23-26] and some substrate binding proteins and $A B C$ transporter have been shown to affect virulence [13, 27-30]. Importantly, PnrA has been shown in two different studies to be immunogenic and protective [20,21], while the impact of the PnrABCD on pathophysiologic pneumococcal processes is unknown. Although in silico analysis (KEGG database) suggested that all enzymes required for purine and pyrimidine synthesis are encoded in the pneumococcal genome, a recent study showed that pneumococci also rely on external nucleosides [31]. The role of PnrA as binding protein for nucleosides has been confirmed by employing 5 -fluorouridine as toxic nucleoside analog [20]. However, which nucleosides are taken up by pneumococci and bind to PnrA was still unknown.

Notably, at this point only few nucleoside ABC-transporters are functionally and structurally well explored. As mentioned, the substrate binding protein PnrA of $S$. pneumoniae has orthologues in other bacteria. These are e.g. PnrA (TmpC) of $T$. pallidum [15], RnsB of S. mutans [17], EF_0176 and EF_0177 of Enterococcus faecalis [32], NupN of B. subtilis [5, 33] and the substrate binding protein BpmA of L. lactis [16, 34]. The transporters or their respective substrate binding proteins exhibit different 
substrate specificities. Nevertheless, all of them transport ribonucleosides and with smaller affinities deoxyribonucleosides, whereas ribose or nucleobases are not bound by these receptors.

In the present study we identified nucleosides as PnrA-binding partners and we monitored nucleoside uptake by pneumococci by the highly accurate ${ }^{1} \mathrm{H}-\mathrm{NMR}$ method. Besides, we performed a complete structural characterization of purine and pyrimidine nucleoside binding by PnrA. Our atomic resolution data revealed how both nucleosides are recognized and explain different experimental binding affinities. Finally, we provide a link between the physiological role of PnrA and pneumococcal virulence.

\section{Results}

\section{Extended logarithmic growth phase of pneumococci in the presence of higher concentrations of nucleosides in vitro.}

For growth and virulence studies we deleted the pnrA gene in D39, its isogenic capsule mutant D39 $\Delta c p s$ and in the bioluminescent D39/ux by insertion deletion mutagenesis [21]. The lack of pnrA expression was indicated by quantitative real-time PCR, which also confirmed gene expression of downstream located pnrBCD genes spd_0740spd_0742 in the parental and pnrA-mutant strains (Figure S1). The loss of a functional PnrA in these strains was indicated by immunoblot analysis using specific anti-PnrA antibodies and flow cytometry, which confirmed the surface localization of PnrA (Figure 1A-C). We further compared the surface abundance of PnrA with another lipoprotein, namely PsaA, which is a manganese binding protein and has been shown to be 
immunogenic like PnrA [35-37]. Indeed, our flow cytometric analysis indicates that PnrA shows a high surface abundance, which exceeds the surface abundance of PsaA clearly as shown recently (Figure $1 \mathrm{~A}$ and $\mathrm{B})$ ([21]. The pnrA- or psaA-mutants did not show any reactivity with the antigen specific antibodies as demonstrated in Figure 1 and thus, these results are in agreement with immunoblotting results published previously [11]. Interestingly, PnrA is also accessible and recognized by anti-PnrA antibodies when we assessed the PnrA surface abundance on the encapsulated wild-type of strain D39 (Figure 1B and C).

We further assessed the effect of loss of function of PnrA under in vitro conditions by culturing the parental D39 strains and the isogenic pnrA-mutants at $37^{\circ} \mathrm{C}$ and $5 \% \mathrm{CO}_{2}$ in chemically-defined medium (RPMImodi) without (Figure 1D) and with supplementation of a $0.5 \mathrm{mM}$ nucleoside mixture (Figure 1D). The encapsulated D39 strain and its isogenic $\Delta p n r A$ mutant reached higher optical densities in both media compared to the non-encapsulated strains. Interestingly, the growth rate was higher in RPMImodi without nucleosides, while higher optical densities could be reached in RPMImodi with nucleosides, which is indicated by an earlier pneumococcal cell lysis or sedimentation of capsule-deficient strains in non-supplemented medium (Figure 1D). Interestingly, no significant difference between the wild-type strain and the isogenic pnrA-mutant was monitored under these conditions in both supplemented and non-supplemented media (Figure 1D). 


\section{Pneumococcal nucleoside consumption.}

Because the analysis of pneumococcal growth in RPMImodi supplemented with nucleosides did not show a significant effect of loss of PnrA function, we measured the effect of PnrA deletion on nucleoside uptake directly. We cultured D39, D39 $\Delta c p s$ and their isogenic $\triangle p n r A$ mutants in $R P M I_{\text {modi }}[12,31]$ individually supplemented with 0.5 $\mathrm{mM}$ of the nucleosides adenosine, guanosine, cytidine, uridine or thymidine. To measure the consumption of nucleosides we took samples from the culture medium during mid-logarithmic phase 5 hours after inoculation $\left(T_{1}\right)$ and from the stationary phase 8 hours after inoculation $\left(\mathrm{T}_{2}\right)$. We finally obtained absolute concentrations of nucleosides by ${ }^{1} \mathrm{H}-\mathrm{NMR}$ spectroscopy. Concentrations of uridine and thymidine in the medium did not decrease during growth (Figure 2D and E), whereas we detected a significant uptake of guanosine, adenosine and cytidine for all strains (Figure 2A - C). The uptake of adenosine (in average $0.30 \mathrm{mM}$ ) and cytidine (in average $0.09 \mathrm{mM}$ ) was similar between the strains and showed no clear difference in the culture of the PnrAdeficient mutant. In contrast, guanosine was taken up by all pneumococcal strains and we monitored significant differences between wild-type and $\Delta p n r A$-deletion mutants for both pairs, the encapsulated and non-encapsulated D39 strain ( $p$-value $<0.01)$. These data suggest that pneumococci metabolize adenosine, cytidine, and guanosine and that loss of function of PnrA affects in particular the consumption of guanosine.

\section{Biochemical analysis of nucleoside-binding to PnrA.}

To assess whether nucleosides bind to PnrA, we tested binding of the nucleosides adenosine, cytidine, guanosine, thymidine, and uridine to PnrA by microscale 
thermophoresis. Both, the nucleosides as ligands and the fluorescently labeled PnrA or the control lipoprotein DacB (L,D-carboxypeptidase [38]) were in solution and we recorded binding of the ligands at $25^{\circ} \mathrm{C}$. All nucleosides bound in a concentration dependent manner to PnrA, but not DacB (Figure 3). The dissociation constants (Kds) were similar for all interactions and in a low $\mu \mathrm{M}$-range (Figure 3). The binding curves showed the best fitting and highest binding activities for guanosine (Kd of $0.29 \times 10^{6}$ $\mu \mathrm{M})$ and cytidine $\left(0.71 \times 10^{6} \mu \mathrm{M}\right)$, followed by adenosine $\left(\mathrm{Kd}\right.$ of $\left.1.71 \times 10^{6} \mu \mathrm{M}\right)$, uridine ( $\mathrm{Kd}$ of $\left.1.81 \times 10^{6} \mu \mathrm{M}\right)$ and thymidine $\left(\mathrm{Kd}\right.$ of $\left.15.7 \times 10^{6} \mu \mathrm{M}\right)$. Thus, binding experiments performed by MST suggest that all tested nucleosides bind to PnrA, which in theory could lead to uptake of the bound nucleoside. However, the molecular details of these interactions were still elusive.

\section{Overall structure of PnrA.}

We solved the crystal structures of PnrA in complex with five nucleosides (adenosine, guanosine, cytidine, thymidine and uridine) with resolutions ranging from 2.20-2.55 $\AA$ (Table S3). The PnrA structural model contains 330 residues including six extra histidines from the His6-tag inserted at the $\mathrm{N}$-terminal part of PnrA for purification purposes. We observed four independent chains in the asymmetric unit of each crystal. The PnrA structure (Figure 4A) presents two domains, the $\mathrm{N}$ domain (residues 2-126 and 270-294) and the $C$ domain (residues $128-268$ and 301-333) both showing a core with six-stranded- $\beta$-sheets surrounded by $\alpha$-helixes (four in the $\mathrm{N}$ domain and five in the $\mathrm{C}$ domain). All the strands in the $\mathrm{N}$-terminal domain are parallel while in the $\mathrm{C}$-terminal domain, five are parallel and one is antiparallel. PnrA, as a member of Cluster B type of 
the Class I substrate binding protein [39], presents the two domains connected by three hinge regions: CR1 (residue 127), CR2 (residue 269) and CR3 (residues 295-300), all of them located in the same area (Figure 4A). The closest structural homologue is the PnrA/TmpC of T. pallidum [15] ( $r m s d$ of $0.80 \AA$ for $247 \mathrm{C} \alpha$ atoms). It is worth mentioning that PnrA in S. pneumoniae has an extra $\beta$-hairpin in the $C$ domain compared to the homologous protein from T. pallidum. The TmpC structure has been reported in complex with three nucleosides adenosine (PDB code 2FQY), guanosine (PDB code 2FQX) and inosine (PDB code 2FQW) describing how these purines bind inside the protein. Most of the substrate-binding proteins present the so-called "Venus-fly-trap" mechanism [40] that involves movement of the $\mathrm{N}$ and $\mathrm{C}$ domains (as rigid bodies) through the hinges (CR1, CR2 and CR3 in PnrA), from an open to a closed conformation, in which the substrate is trapped between both domains. All our structures were obtained in complex with a nucleoside and thus, represent the closed conformation structures. Our trials to get crystals of PnrA without nucleosides (open conformation) failed, likely due to the dynamic behavior of the two domains in the absence of substrate. However, structural comparison with the open conformation of ALBP from E. coli (periplasmic binding protein for the allose system, PDB code 1GUB), a substrate-binding protein of the same group and Cluster type, allows extrapolation of the changes suffered upon substrate binding (Figure S2). Superimposition of the Ndomains in both structures indicates that large displacements $(\sim 16 \AA)$ and rotation of the C domain is needed to trap the substrate in PnrA. Consistently, our crystallographic complexes reveal that the binding site in PnrA is located between the $\mathrm{N}$ and $\mathrm{C}$ domains (Figure 4A) and residues from each domain participate in substrate recognition. The 
nucleoside was found in each of the four independent monomers of the asymmetric unit of the five complexes (20 independent complexes in total) that, together with the excellent, initial and refined, electron-density maps (Figure S3 and Figure S4) allowed a very precise description of nucleoside recognition by pneumococcal PnrA (Figure 4B-F).

\section{Molecular basis for nucleoside recognition by PnrA.}

We crystallized five different nucleosides from three different types in complex with PnrA: purine ribonucleosides (adenosine and guanosine), pyrimidine ribonucleosides (cytidine and uridine), and pyrimidine deoxyribonucleosides (thymidine). This is, to our knowledge, the first example of a nucleoside substrate-binding protein in complex with both pyrimidine and purine bases and with both the ribose- and deoxyribosenucleosides.

Overall the structures of the PnrA:nucleoside complexes are very similar with rmsd values for backbone superimpositions around $0.25 \AA$ between them. Our structural comparison (Figure S5A) shows that backbone changes are concentrated in loops 27 36 and 64-68, both from the $\mathrm{N}$-terminal domain. Complex 3 (PnrA:cytidine) presents the most variable structure (Figure S5B). In complex 3 twist in the peptide bond Thr29Gly30 results in a significant displacement of the loop 27-36 moving away from the nucleoside-binding site. In our complexes all nucleosides are placed in the same orientation into the PnrA binding site and several van der Waals and $\mathrm{H}$-bond interactions with residues from the two domains are observed; a network of conserved water molecules provides further stabilization of the nucleoside in the binding site (Figure 4BF and Figure S6). Interestingly, all nucleosides are sandwiched by two aromatic 
residues (Phe37 and Phe187) coming from each domain of PnrA. These van der Waals interactions are complemented by many $\mathrm{H}$-bond interactions also coming from the two PnrA domains. According to donor-acceptor distances three types of $\mathrm{H}$-bond interactions can be distinguished [41]: strong or mostly covalent (2.20 to $2.50 \AA)$, moderate or mostly electrostatic ( 2.51 to $3.20 \AA$ ) and weak or electrostatic ( 3.21 to 4.00 $\AA$ A). Figure 5 shows the type of $\mathrm{H}$-bond between PnrA and nucleosides observed in the five PnrA:nucleoside complexes. Interestingly, relevant differences are detected among them, allowing identification of the singularities in their recognition by PnrA. The complete numerical values for the $\mathrm{H}$-bond distances are listed in Table S4. Adenosine (complex 1) and guanosine (complex 2) nucleosides present the same binding pattern with several key residues from both the $\mathrm{N}$ - and $\mathrm{C}$-terminal domains involved (Figure 4B-C). The nitrogenous base is widely stabilized by stacking interactions by Phe187 and Phe37 and by polar interactions through side chains of Asp28 and Asn38, and main chain atoms from Thr29 and Gly30. According to distances, $\mathrm{H}$-bonds between purine nucleosides and Asp28 and Asn38 have a moderate character (Figure 5). The presence of more donor/acceptor groups in guanosine, allows a larger number of $\mathrm{H}$-bonds with PnrA than in adenosine (Figure 5). Concerning the ribose moiety, four residues (Asp112, Lys268, Asp242 and the Gly213 backbone) establish polar interactions with the saccharide hydroxyl groups. It is worth to mention the role of Asp112 (from $\mathrm{N}$ domain) that makes a salt-bridge interaction with Lys268 (from C domain) linking the two PnrA domains and also establishing a strong $\mathrm{H}$ bond with the 2 '-hydroxyl group of the ribose cycle (Figure 4B-C and Figure 5). Lys268 and Asp242 play a central role by making four $\mathrm{H}$-bonds with the hydroxyl groups of 
ribose. While all these polar interactions are performed by proper orientation of the side chains of the involved residues, Gly213 establishes a H-bond with the $5^{\prime}$-hydroxyl group through its backbone $\mathrm{N}$ atom (Figure 4B-C). A total of nine (adenosine) or ten (guanosine) $\mathrm{H}$-bond interactions are observed in their respective complexes with PnrA (Figure 5).

For pyrimidine ribonucleosides, cytidine (complex 3 ) or uridine (complex 4), recognition of the ribose moieties presents the same interaction pattern like adenosine and guanosine complexes (Figure 4) and thus, the four complexes have the ribose moieties in the same position (Figure S5B). However, stronger interactions are observed by Asp112 in the adenosine and guanosine cases compared to cytidine or uridine (Figure 5). As expected, we observed differences in the recognition of the pyrimidine (complexes 3 and 4) versus purine (complexes 1 and 2) moieties (Figure 4 and Figure 5). Conformation of loop 27-36 changes (i) to adapt to purine or pyrimidine moieties (up to $1.4 \AA$ difference among $\mathrm{C} \alpha$ backbones between adenosine/guanosine and thymidine/uridine complexes) and (ii) to establish $\mathrm{H}$-bond interactions with $\mathrm{N}$ or $\mathrm{O}$ atoms at position 6 (Figure 4E and Figure 5). It is worth to mention that cytidine complex, presents a different conformation for loop 27-36 compared with that of the other pyrimidine complexes (Figure S5B). Only in this case the side-chain of Asp28 reorients its carboxylate moiety to establish a double $\mathrm{H}$-bond with $\mathrm{N}$ atoms at positions 3 and 4 (Figure 4 and Figure 5) altering conformation of loop 27-36. Thus, plasticity in loop 2736 is relevant in nucleoside recognition; in this sense it is worth to mention that Ser36 located at the hinge of the loop is observed systematically as a Ramachandran outlier (see methods). 
As observed in the pyrimidine ribonucleoside group, cytidine and uridine have the same number of moderate $\mathrm{H}$-bonds, although cytidine presents two weak interactions more (Figure 5).

For thymidine (pyrimidine deoxyribonucleoside) (Figure 4F), the interaction pattern is similar to that of uridine (Figure 4E), but without the strong interactions by Asp112 and Lys268 with the hydroxyl group at 2' that is absent in deoxyribonucleosides. The methyl group of thymidine is trapped by van der Waals interactions with the neighbors Ca from Gly213 and the C $\beta$ groups from Phe37 and Phe187 but this nucleoside presents a lower number of interactions and some conserved moderate interactions turn into weak ones for thymidine (Figure 5). In conclusion, thymidine is the worst recognized nucleoside with only seven interactions and three of them are classified as weak.

Our structural comparison of the adenosine and guanosine recognition in PnrA and in its homologue TmpC [15] reveals a similar pattern in both cases with similar residues and water molecules involved (Figure S7). Main differences concern the side-chain conformation of Asp27 (Asp28 in PnrA) and the number of water molecules involved in nucleoside stabilization. As observed in PnrA plasticity in the loop 27-36 (26-36 in $\mathrm{TmpC}$ ) is required to adapt to each nucleoside. It is worth to mentioning the proteinnucleoside interactions found in the TmpC:inosine complex (Figure S7C) follows the recognition pattern found in adenosine and guanosine.

\section{Impact of PnrA on phagocytosis in murine macrophages.}

To investigate whether the deficiency of PnrA affects uptake or intracellular survival of pneumococci by professional phagocytes, we infected murine macrophages J774A.1 
with $\mathrm{D} 39 \Delta c p s$ and its isogenic pnrA-mutant $\mathrm{D} 39 \Delta c p s \Delta p n r A$. We determined the number of intracellular and recovered bacterial survivors by killing extracellular pneumococci 30 min post infection by antibiotic treatment. Plating of the intracellular bacteria revealed a significantly but not dramatically higher number of recovered D39 $\Delta c p s \Delta p n r A$ pneumococci compared to the parental strain (Figure 6A). To assess the intracellular fate of pneumococci, we performed infection kinetics by recovering pneumococci at different time points of phagocytosis. The data show that intracellular survival of wildtype and PnrA-deficient pneumococci was comparable, indicated by a similar decrease in numbers of viable and recovered pneumococci with a reduction of approximately $50 \%$ within $2 \mathrm{~h}$. Similar to these data, our quantification of pneumococci associated with macrophages or located intracellularly conducted by double immunofluorescence microscopy, did not show a clear difference between D39 $\Delta c p s$ and its isogenic pnrAmutant D39 $\Delta c p s \Delta p n r A$ (Figure 6C and D; Figure S8). Interestingly, the fluorescence microscopy suggested, similar to the antibiotic protection assays, that the number of extracellular bacteria bound to phagocytes decreased due to their uptake within 1 to 2 hours post-infection (Figure 6D). These data suggest that loss of function of PnrA does not clearly affect survival or killing after phagocytic uptake by J774.1 macrophages (Figure 6B).

\section{Influence of PnrA on lung infections in the pneumococcal acute pneumonia mouse model.}

To investigate the impact of PnrA and nucleoside uptake under pathophysiological conditions, we compared the virulence of the pnrA-mutant to wild-type pneumococci in 
the acute pneumonia and sepsis mouse infection models. To analyze the impact of PnrA on pneumococcal survival in the blood, mice $(n=12)$ were challenged intraperitoneally with $5 \times 10^{3} \mathrm{CFU}$ S. p. D39/ux or its isogenic $\Delta p n r A$-mutant (Figure 7B). No significant differences between wild-type and pnrA-mutant infected mice could be observed indicating that pnrA has no impact on the development of septicemia and survival of pneumococci in the blood stream.

In the acute pneumonia model mice $(n=10)$ were intranasally infected with $5 \times 10^{7} \mathrm{CFU}$ of D39/ux or its isogenic $\Delta p n r A$-mutant (D39/ux $\Delta p n r A)$. Twenty-four hours post-infection wild-type infected mice developed pneumonia, while bioluminescence, as a sign of high bacterial load in mice infected with PnrA-deficient pneumococci, could be clearly monitored $32 \mathrm{~h}$ post-infection (Figure $7 \mathrm{C}$ ). Survival of mice infected with the pnrAmutant was moderately but not significantly improved compared to wild-type infected mice (Figure 7A). However, the infection process was not altered in mice infected with the pnrA-mutant, because these mice invariably developed pneumonia followed by septicemia. Thus the dissemination was quite similar to wild-type infected mice. Taken together, these in vivo data suggest that the deficiency of PnrA at least partially attenuates pneumococcal fitness, although this could not be finally demonstrated when mice were separately infected with the wild-type or mutant.

\section{PnrA-deficiency diminishes the efficiency of nasopharyngeal colonization by pneumococci}

The acute pneumonia model with separately infected mice suggested that the pnrAmutant is slightly attenuated under in vivo conditions (Figure 7A). To demonstrate this 
effect more clearly, we assessed the different efficiency of bioluminescent D39/ux and D39/ux $\Delta p n r A$ to colonize CD-1 outbred mice in a co-infection experiment. Mice $(n=14)$ were co-infected intranasally with a 1:1 ratio of the pnrA-mutant and the isogenic wildtype D39/ux. After $24 \mathrm{~h}$ and $48 \mathrm{~h}$, respectively, we recovered pneumococci from the nasopharynx, bronchi and blood and determined the CFU to calculate the ratio of pnrAmutant vs. wild-type bacteria recovered from each individual mouse represented by the competitive index $(\mathrm{Cl})$. While there was no clear difference in the nasopharynx after 24 $\mathrm{h}($ mean $\mathrm{Cl}=1.03)$, the wild-type D39/ux out-competed the pnrA-mutant in the deeper airways at $24 \mathrm{~h}$ (mean $\mathrm{Cl}=0.665$ ) post-infection (Figure 7D). The difference was much clearer $48 \mathrm{~h}$ post-infection. In both host compartments, the nasopharynx and bronchi, the wild-type had out-competed the pnrA-mutant resulting in a competitive index $(\mathrm{Cl})$ below 1 , with a mean of 0.765 and 0.44 . Furthermore, we determined the bacterial load in the blood after $24 \mathrm{~h}$ and $48 \mathrm{~h}$. Twenty-four h post-infection, only 3 out of 14 mice showed pneumococci in the blood (mean $\mathrm{Cl}=0.741$ ), while $48 \mathrm{~h}$ post-infection all mice had a high bacterial load in the blood (mean $\mathrm{Cl}=0.004)$. As indicated by the $\mathrm{Cl}$, exclusively wild-type but not PnrA-deficient pneumococci entered the bloodstream. In conclusion, the co-infection experiments clearly show the lower fitness of the pnrAmutant under in vivo conditions suggesting that PnrA is needed for full virulence.

\section{Discussion}

The involvement of PnrA in the uptake of extracellular nucleosides by $S$. pneumoniae as a substrate binding protein has recently been reported [20], but the exact substrates and mechanism of uptake have not yet been investigated. In the present study, we 
show that the highly conserved and surface abundant lipoprotein PnrA has, in general, the highest affinity to bind purine-based nucleosides such as adenosine and guanosine, rather than pyrimidine-based nucleosides like cytidine, uridine and thymidine. In an effort to provide the molecular basis of such selectivity, high-resolution crystal structures of PnrA in complex with purine-based nucleosides (adenosine, guanosine) and pyrimidine-based nucleosides (cytidine, uridine and thymidine) have been solved. This is, to the best of our knowledge, the first example of a nucleoside substrate-binding protein in complex with both pyrimidine and purine bases and with both the ribose- and deoxyribose-nucleosides. Crystal structures unveil a clear pattern of interactions in which both the $\mathrm{N}$ - and $\mathrm{C}$ - domains of PnrA contribute. In all complexes the ribose moiety is strongly recognized through a conserved network of $\mathrm{H}$-bond interactions (by Asp112, Lys268, Asp242 and Gly213), while the single or double heterocyclic compound of the nitrogenous bases is recognized through $\mathrm{H}$-bond interactions by residues from loop 27 36 and by Asn38. Plasticity in loop $27-36$ is essential to adapt differences between purine- and pyrimidine-based nucleosides. In-depth analysis of $\mathrm{H}$-bond interactions (Figure 5 and Table S4) indicates that, despite the conserved interaction pattern, all the nucleosides present differences in the number of interactions and/or in their strength. Guanosine shows the largest number of interactions and the strongest ones, explaining why it has the highest affinity in the MST binding experiments (Figure 3). The same number (ten) of interactions was observed for cytidine, the next nucleoside in the affinity ranking by MST. However, the strong interaction between Asp112 and O2' observed for guanosine is replaced in cytidine by a moderate $\mathrm{H}$-bond (Figure 5) (mean value for the Asp112-O2' interaction in the cytidine complex is $2.69 \AA$ versus $2.51 \AA$ in the guanosine 
complex (Table S4)). Interestingly, the weakened Asp112-O2' interaction in cytidine is partially compensated by stronger interaction with the nitrogenous base through Asp28 that reorients its carboxylate moiety to establish a double $\mathrm{H}$-bond with $\mathrm{N}$ atoms at positions 3 and 4 (Figure 4D). This double bond by Asp28 is observed only in the cytidine complex (Figure 4D) that, together with the number of interactions, explains why the binding affinity observed by MST is better in cytidine than in adenosine. Adenosine and uridine nucleosides show both nine $\mathrm{H}$-bond interactions (Figure 5). However, one of them (Asp112-O2') is strong in adenosine but moderate in uridine, which is in agreement with the small decrease in binding affinity for uridine (Figure 3). Finally, thymidine shows the lowest number of $\mathrm{H}$-bond interactions and the weaker ones, thus, explaining the observed lowest affinity by PnrA. In summary, both MST and crystallographic results provide a detailed description of nucleoside recognition by PnrA at the molecular level.

The relevance of PnrA in pneumococcal growth and substrate specificity upon pnrAdeletion has also been studied. The D39 $\Delta p n r A$ strain did not show significant changes in growth behavior compared to the wild-type in THY (data not shown) or in chemically defined medium RPMlmodi. Likewise, no changes in growth behavior of homologous proteins in other bacteria were observed after the deletion of RnsB of $S$. mutans [17] and EF_0176 and EF_0177 of Enterococcus faecalis [32]. Interestingly, pneumococcal growth behavior changed following the addition of $0.5 \mathrm{mM}$ of the nucleosides mixture. Pneumococci were able to grow for a longer period of time and there was a clear delay in reaching the stationary phase compared to growth in RPMImodi without the addition of nucleosides (Figure 2). It is well known that nucleosides 
play an important role in cell physiology. With approximately $2-15 \mu \mathrm{M}$ the average concentration of nucleosides in extracellular fluids and cells is in the lower micromolar range [42]. However, measuring nucleoside concentrations is hampered by their rapid cellular uptake and degradation [43]. Nucleosides are used as precursors for nucleotides, as activators of precursors, as constituents of coenzymes and play a role in regulation of many cellular processes [1]. In fact, the presence of the key role players "nucleosides" out- or inside pneumococci seems to change the physiological status of the pneumococcus. We measured uptake of nucleosides and indicated a role of PnrA at least for guanosine import. Thus, the available concentration in host compartments, the binding affinity, high surface abundance and substrate specificity might determine uptake of guanosine via PnrA. Our nucleoside uptake data are also in agreement with previous data [20] and strengthen the idea that other nucleoside uptake systems have to be produced by pneumococci for uptake of the other nucleosides. This is illustrated by the fact that uptake of adenosine and cytidine is not affected by the loss of function of PnrA. The additional nucleoside transport systems might be structurally different from PnrA, because we could not find a structural homologue to PnrA by bioinformatic analysis.

The crucial role of uptake systems and lipoproteins for pneumococcal fitness and full virulence has been shown earlier [44-47]. Other striking examples are the glutamine transporters [13], zinc and manganese Psa transport systems [27]. Similar to the above mentioned transport systems, but not associated with such a dramatic loss of physiological fitness and loss of virulence observed for the above mentioned transport systems, the inactivation of the Pnr transport system led to attenuated pneumococci. In 
vitro experiments with macrophages did not demonstrate a significantly enhanced or reduced phagocytosis of PnrA-deficient pneumococci, when both the antibiotic protection assays and the number of intracellular pneumococci determined by fluorescence microscopy were considered. Thus, we are at this stage unable to evaluate the role of PnrA in the interaction with phagocytes and additional experiments with primary antigen presenting cells are required. In addition, the pnrA-mutant did not show any differences in virulence in the sepsis mouse infection model. In contrast, the intranasal infection with $\triangle p n r A$ pneumococci resulted in a delay of developing a severe lung infections in the acute pneumonia model compared to the isogenic wild-type when independent mouse groups were used for infection. The attenuation of the pnrA-mutant was more clearly demonstrated in mouse co-infection experiments, in which the wildtype out-competed the mutant in the nasopharynx and lower respiratory tract. The attenuation of the pnrA-mutant was confirmed when analyzing the bacterial load in the blood. Only wild-type pneumococci were found in the blood suggesting that the deficiency of PnrA hampers pneumococci to enter the bloodstream when competing with the isogenic wild-type.

In conclusion, we show for the first time the complex structures of a nucleoside-binding protein in complex with both purine and pyrimidine nucleosides providing a detailed molecular picture of the interaction between a binding protein and its substrate. The complex structures are in agreement with biochemical analyses and nucleoside consumption. Finally, the relevance of this Pnr transport system for full pneumococcal 
fitness is shown under in vivo conditions demonstrating that in addition to the de novo synthesis the uptake of nucleosides under in vivo conditions is essential.

\section{METHODS}

\section{Bacterial strains, plasmids and culture conditions.}

E. coli and S. pneumoniae strains, and plasmids used in this study are listed in Table S1. E. coli were cultured on Luria-Bertani (LB) plates or in LB broth, supplemented with ampicillin $(100 \mu \mathrm{g} / \mathrm{ml})$, erythromycin $(250 \mu \mathrm{g} / \mathrm{ml})$ or kanamycin $(50 \mu \mathrm{g} / \mathrm{ml})$ depending on the plasmid. Transformation of E. coli with plasmid DNA was performed using $\mathrm{CaCl}_{2}$ competent cells. S. pneumoniae and isogenic mutants of serotype 2 strain D39 (NCTC7466) were grown on blood agar plates (Oxoid, Wesel, Germany), cultured in Todd-Hewitt broth (Oxoid, Basingstoke, England) supplemented with $0.5 \%$ yeast extract (THY; Roth, Karlsruhe, Germany) or in RPMI1640 (HyClone, Germany) supplemented with $30.5 \mathrm{mM}$ glucose, $0.65 \mathrm{mM}$ uracil, $0.27 \mathrm{mM}$ adenine, $1.1 \mathrm{mM}$ glycine, $0.24 \mathrm{mM}$ choline chloride, $1.7 \mathrm{mM} \mathrm{NaH}_{2} \mathrm{PO}_{4} \cdot \mathrm{H}_{2} \mathrm{O}$, $3.8 \mathrm{mM} \mathrm{Na}_{2} \mathrm{HPO}_{4}$ and $27 \mathrm{mM} \mathrm{NaHCO}_{3}$ referred to as chemically-defined medium (CDM or RPMImodi medium) [12, 48]. Cultivation of pneumococci was conducted at $37^{\circ} \mathrm{C}$ and $5 \% \mathrm{CO}_{2}$, and liquid cultures were grown without agitation to mid-log phase ( $A_{600} 0.35$ to 0.4 ). The construction of the mutant D39 $\Delta p n r A$ was described previously [21] and bioluminescent D39/ux $\Delta p n r A$ were constructed by transforming D39/ux with the pnrA-mutagenesis plasmid (Table S1). 


\section{PnrABCD Gene expression analysis}

Total RNA was isolated from strain D39 $\Delta c p s$ and the corresponding pnrA-mutant as described [49]. Briefly, bacteria were grown in THY medium up to early logarithmic growth phase $\left(\mathrm{OD}_{600 \mathrm{~nm}}=0.35\right)$ and $15 \mathrm{ml}$ killing buffer $\left(0.2 \mathrm{M}\right.$ Tris, $\mathrm{pH} 7.5,0.1 \mathrm{M} \mathrm{MgCl}_{2}$, $0.2 \mathrm{M} \mathrm{NaN}_{3}$ ) were added to $30 \mathrm{ml}$ culture and harvested by centrifugation at $4000 \mathrm{xg}$ for 10 min at $4^{\circ} \mathrm{C}$. Bacterial sediment was immediately frozen in liquid nitrogen and stored at $-80^{\circ} \mathrm{C}$. The RNA preparation was carried out with the RNA isolation kit from Roboklon following the bacterial RNA purification protocol. In addition the RNA preparation was treated with TURBO ${ }^{\mathrm{TM}}$ DNase (Invitrogen) for $30 \mathrm{~min}$ at $37^{\circ} \mathrm{C}$ followed by RNA purification with the RNA clean up and concentration kit from Norgen Biotek Corp. as recommended by the supplier. For cDNA synthesis $1 \mu \mathrm{g}$ total RNA was incubated with hexameric primers (1:5 diluted) from Fisher Scientific at $65^{\circ} \mathrm{C}$ for $5 \mathrm{~min}$, cooled down to $50^{\circ} \mathrm{C}$ before the Superscript transcriptase III (Invitrogen) was added. cDNA synthesis was carried out for $60 \mathrm{~min}$ and the reaction was inactivated at $70^{\circ} \mathrm{C}$ for $15 \mathrm{~min}$.

For quantitative real-time PCR ( $\mathrm{qPCR}$ ) relative standard curves were determined with cDNA received from D39 $\triangle$ cps. For the qRT-PCR analysis primers were selected for the pnrA gene (spd_0739) and the following three downstream genes (spd_0740 to spd_0742). As control the enolase gene (spd_1012) was selected. The primers used in this study are listed in Table S2. The qRT-PCR reaction was carried out with the StepOnePlus thermocycler (Applied Biosystems) using the iTaq Universal SYBR Green Supermix from BioRad. The qRT-PCR reaction steps were as followed: $95^{\circ} \mathrm{C}$ for 2 min (initial denaturation), then $95^{\circ} \mathrm{C}$ for $15 \mathrm{sec}$, followed by $60^{\circ} \mathrm{C}$ for $30 \mathrm{sec}$ and synthesis at $72^{\circ} \mathrm{C}$ for $30 \mathrm{sec}$ for 40 cycles. Each reaction was carried out three times. Ct values were 
determined as well as the $\Delta \Delta C T$ ( $\log _{2}$ of the fold change of expression) values for differential gene expression and comparison of wild-type and the pnrA-mutant. The calculations were made by the Pfaffl method [50]. Gene expression analysis indicated that pnrBCD gene expression is not affected by insertion deletion mutagenesis.

\section{Heterologous expression and purification of PnrA.}

PnrA (SP_0845 in TIGR4) was heterologously expressed in E. coliBL21 and purification was performed as described [21]. For structural analysis and functional experiments the His6-tag of His6-PnrA the purified fusion protein was removed by treatment with TEV protease, and separated from rPnrA protein by a re-purification step using the HisTrap ${ }^{\mathrm{TM}} \mathrm{HP}$ Ni-NTA column. The highly pure, tag-free protein was used for the generation of antisera in outbred CD-1 ${ }^{\circledR}$ mice against rPnrA [21]. Briefly, mice were intraperitoneally immunized and boosted two times with $30 \mu \mathrm{g}$ of rPnrA mixed 1:1 with incomplete Freund's adjuvant. The purity of the protein was visualized by Coomassie Brilliant Blue (CBB) stained SDS-PAGE and by immunoblot analysis using mouse antiPnrA specific antiserum.

\section{Growth behavior of pneumococcal strains.}

Growth behavior of the parental pneumococcal strain D39 and its isogenic mutants was investigated in RPMImodi medium with and without supplementation of $0.5 \mathrm{mM}$ of nucleosides: adenosine, guanosine, cytidine, uridine and thymidine (SigmaAldrich,Germany). The overnight culture (10 h) of pneumococci on blood agar plates 
was used to inoculate $40 \mathrm{ml}$ of RPMImodi to reach the starting $\mathrm{OD}_{600} \sim 0.1$ followed by incubation at $37^{\circ} \mathrm{C}$ without agitation. The optical density was measured every hour.

\section{Flow cytometric analysis of PnrA and PsaA surface abundance.}

The abundance of PnrA and PsaA, which is a substrate binding protein for manganese, was analyzed by flow cytometry using specific anti-PnrA or PsaA mouse polyclonal IgG antibodies as described [11, 21]. Briefly, pneumococci were cultured in $30 \mathrm{ml} \mathrm{THY} \mathrm{to}$ A600 0.35-0.4 and after washing PBS and resuspension in PBS $2 \times 10^{8}$ pneumococci were incubated with the antigen specific polyclonal IgG antibodies. After washing the bacteria were stained for 45 min at $4^{\circ} \mathrm{C}$ with secondary antibody goat anti-mouse $\lg \mathrm{G}$ coupled Alexa-Fluor-488 (1:1000, Invitrogen). Bacteria were then washed again and fixed with $1 \%$ paraformaldehyde over night at $4^{\circ} \mathrm{C}$. Flow cytometry was conducted with the FACSCalibur ${ }^{\mathrm{TM}}$ (BD Biosciences, Heidelberg, Germany) and the CellQuestPro Software 6.0 (BD Biosciences) was used for data acquisition while analysis of the data was performed with Flowing Software 2.5.1 (by Perttu Terho, Turku Centre for Biotechnology).

\section{Monitoring the nucleosides uptake by ${ }^{1} \mathrm{H}-\mathrm{NMR}$ analysis.}

Two ml samples of cell-free pneumococci cultured in RPMImodi supplemented with 0.5 $\mathrm{mM}$ nucleosides were obtained by filtration and directly frozen until measurement by ${ }^{1} \mathrm{H}$ NMR. Two time points of the bacterial growth were taken: mid-logarithmic phase after 5 hours of inoculation $\left(T_{1}\right)$ and stationary phase after 8 hours of inoculation $\left(T_{2}\right) .{ }^{1} \mathrm{H}-\mathrm{NMR}$ analysis was carried out as described previously [51]. Briefly, $400 \mu \mathrm{l}$ of each sample 
was mixed with $200 \mu \mathrm{l}$ of a sodium hydrogen phosphate buffer $(0.2 \mathrm{M}, \mathrm{pH} 7.0)$ to avoid chemical shifts due to $\mathrm{pH}$, which was made up with $50 \% \mathrm{D}_{2} \mathrm{O}$. The buffer also contained $1 \mathrm{mM}$ trimethylsilyl propanoic acid (TSP), which was used as a reference signal at 0.0 ppm. To obtain NMR spectra a 1D-NOESY pulse sequence was used with 64 FID scans with $600.27 \mathrm{MHz}$ at a temperature of $310 \mathrm{~K}$ using a Bruker AVANCE-II $600 \mathrm{NMR}$ spectrometer operated by TOPSPIN 3.2 software (both from Bruker Biospin). For qualitative and quantitative data analysis AMIX (Bruker Biospin, version 3.9.14) was used. Signals were identified and annotated using reference substances and spectra from HMDB. Absolute quantification was performed by normalizing the NMR chemical shift signal of the metabolite on the area of an Bruker ERETIC2 signal calculated using the PULCON approach [52].

\section{Immunoblot analysis.}

For immunoblot analysis pneumococcal cell lysates $5 \times 10^{8}$ bacteria were loaded per lane. Proteins were separated by SDS-PAGE and transferred onto a nitrocellulose membrane by semidry blotting (Bio-Rad Laboratories, Munich, Germany). The membrane was blocked with 5\% skim milk (Roth) in Tris buffered saline (TBS).

Detection of PnrA was conducted with specific mouse anti-PnrA antibodies and goat anti-mouse IgG horseradish peroxidase conjugate (Dianova, Hamburg, Germany; 1:5000) as described [21]. The loading control protein enolase was detected with rabbit anti-enolase antibodies and a goat anti-rabbit IgG horseradish peroxidase conjugate (1:5000). Enhanced chemiluminescence (luminol and p-coumaric acid, Roth) was used for signal detection. 


\section{Microscale thermophoresis.}

The PnrA protein was labeled using the Protein Labeling Kit RED-NHS $2^{\text {nd }}$ generation (NanoTemper Technologies). The labeling reaction was performed according to the manufacturer's instructions in the supplied labeling buffer applying a concentration of 20 $\mu \mathrm{M}$ protein (molar dye:protein ratio $\approx 3: 1$ ) at room temperature for 30 min in the dark. Unreacted dye was removed with the supplied dye removal column equilibrated with MST buffer (50 mM Tris $\mathrm{HCl} \mathrm{pH} 7.8,150 \mathrm{mM} \mathrm{NaCl}, 10 \mathrm{mM} \mathrm{MgCl}$ ). The degree of labeling was determined using UV/VIS spectrophotometry at $650 \mathrm{~nm}$ and $280 \mathrm{~nm}$. A degree of labeling of 0.8 was typically achieved. The labeled PnrA protein was adjusted to $3.33 \mu \mathrm{M}$ with MST buffer supplemented with $0.05 \%$ Tween 20 . The ligands (adenosine, guanosine, cytidine, thymidine and uridine) were dissolved in MST buffer supplemented with $0.05 \%$ Tween 20 , and a series of $161: 1$ dilutions was prepared using the same buffer, producing ligand concentrations ranging from $200 \mu \mathrm{M}$ to 0.0244 $\mu \mathrm{M}$. For the measurement, each ligand dilution was mixed with one volume of labeled PnrA, which led to a final concentration of $20 \mathrm{nM}$ PnrA and final ligand concentrations ranging from $100 \mu \mathrm{M}$ to $0.0122 \mu \mathrm{M}$. The samples were loaded into Monolith NT.115 Premium Capillaries (NanoTemper Technologies). MST was measured using a Monolith NT.115 instrument (NanoTemper Technologies) at an ambient temperature of $25^{\circ} \mathrm{C}$. Instrument parameters were adjusted to $80 \%$ LED power and medium MST power. Data of three independently pipetted measurements were analyzed (MO.Affinity Analysis software version 2.3, NanoTemper Technologies) using the signal from an MST- on time of $15 \mathrm{~s}$. 


\section{Protein crystallization.}

PnrA was concentrated to $10 \mathrm{mg} / \mathrm{ml}$ in a $20 \mathrm{mM}$ TRIS buffer at $\mathrm{pH} 8.2$ while all solid nucleosides were diluted in water in a 40 or $50 \mathrm{mM}$ solution. The five complexes were obtained by the co-crystallization method, adding the nucleoside to the protein solution in a final concentration of $5 \mathrm{mM}$. Crystals were obtained by the vapor-diffused hangingdrop method mixing $2 \mu$ protein nucleoside mix with $1 \mu$ precipitant solution. Precipitant solution was formed by $28 \%$ polyethylene glycol $600,0.2 \mathrm{M}$ calcium acetate and $0.1 \mathrm{M}$ sodium cacodylate ( $\mathrm{pH}$ 6.5). Drops were equilibrated against $500 \mu \mathrm{l}$ of the precipitant in the reservoir chamber. As $28 \%$ polyethylene glycol 600 acts as cryo-protectant, thus crystals were directly flash-cooled in liquid nitrogen.

\section{X-Ray diffraction data collection and structure determination.}

All data sets were collected at $100 \mathrm{~K}$ with PILATUS 6M detector on beamline XALOC of the ALBA Synchrotron facility (Barcelona, Spain). Crystals for PnrA:nucleoside complexes belong to the orthorhombic C 2221 space group with four molecules in the AU. Specific experimental details for each of the five complexes are detailed in Table S3. Data processing and scaling were accomplished with XDS [53] and AIMLESS [54]. The structures were solved by the molecular replacement method by using the structure of PnrA from Treponema pallidum (PDB code 2FQW) as the search model (92\% sequence cover with $39 \%$ identity). The rotational and translational searches were performed using PHASER [55]. The models were then subjected to iterative cycles of model building and refinement with COOT [56] and PHENIX [57]. The complete PnrA structure together with the His6-tag, used for purification purposes, were seen in the 
electron density map. Overall, the final model presents good statistics and geometry (Table S3). While Ramachandran outliers corresponds mainly to some His residues form the His6-tag, it is worth to mention that Ser36, located at the hinge of the binding loop 27-36, was also observed as a Ramachandran outlier pointing to a physiological role in this outlier (see text for details).

\section{Phagocytosis experiments and immunofluorescence microscopy.}

To determine the rate of phagocytosed wild-type and mutant pneumococci and their intracellular survival in macrophages, phagocytosis experiments with J774A.1 murine macrophages (DSMZ, Braunschweig, Germany) were carried out as described previously $[13,58]$. Briefly, confluent monolayers of $\mathrm{J} 774$ cells $\left(2 \times 10^{5}\right)$ cultured in RPMI 1640/10\% fetal bovine serum (FBS; HyClone, Germany) were incubated for 30 min with pneumococci (D39 $\Delta c p s$, D39 $\Delta c p s \Delta p n r A)$. Extracellular bacteria and non-adherent pneumococci were killed by antibiotic protection assay. The infected cells were washed three times with infection medium (RPMI 1640/1\% FBS) and then replaced and incubated for $1 \mathrm{~h}$ with RPMI 1640/1\% FBS containing gentamicin $(100 \mu \mathrm{g} / \mathrm{ml})$ and penicillin $\mathrm{G}$ (100 units/ml) to kill non-internalized bacteria. Intracellular pneumococci were recovered by saponin-mediated lysis $(1 \% \mathrm{w} / \mathrm{v})$ of macrophages [58] and the number of recovered and viable pneumococci (survivors) was determined by quantitative plating of the released intracellular pneumococci on blood agar plates (Oxoid, Germany). Experiments were conducted four times in duplicates. Fluorescence microscopy was performed to visualize attached or internalized pneumococci. Macrophages were seeded on glass coverslips ( $12 \mathrm{~mm}$ ) in wells of a 24-well cell 
culture plate and infected after two days with pneumococci as described above. Postinfection unbound bacteria were removed by washing and infected host cells were fixed with $1 \%$ paraformaldehyde. Double immunofluorescence staining was carried out as described previously [58]. Image acquisition was performed with a fluorescence microscope (Zeiss Axio-Observer.Z1 with VisiGrid, Coolsnap HQ and Visiview Imaging software (Visitron Systems GmbH, Puchheim, Germany). Each bar in the images represents $10 \mu \mathrm{m}$. All experiments were performed four times in duplicate.

\section{Acute pneumonia and sepsis mouse models.}

Pneumococci were cultured to an $\mathrm{OD}_{600}$ of 0.35 in $40 \mathrm{ml}$ THY-medium with $10 \%$ heatinactivated FBS. Bacteria were sedimented and resuspended in $1 \mathrm{ml}$ PBS supplemented with $1.0 \%$ FBS. Samples were diluted to infect eight to ten weeks old female CD1 outbred mice (Charles River, Sulzfeld, Germany) with $5 \times 10^{7}$ CFU per 10 $\mu l$ In the acute pneumonia infection model mice were anesthetized by intraperitoneal injections of ketamine (Ketanest S; Pfizer Pharma, Karslruhe, Germany) and $2 \%$ xylazine (Rompun ${ }^{\circledR}$; Provet AG, Lyssach, Germany). Ten mice per group were intranasally infected with either the parental D39/ux strain or the isogenic mutant D39/ux $\Delta$ pnrA as described previously $[59,60]$. The infection was monitored in real time using the IVIS ${ }^{\circledR}$ Spectrum imaging system (Caliper Life Sciences) $[13,58]$. The mice group which is infected with the parental strain D39/ux is identical to the group that has been published earlier [38], because the infection experiments were performed in parallel to adhere to the $3 \mathrm{R}$ principle. For the sepsis mouse model eight to ten weeks old female CD-1 mice $(n=12)$ were intraperitoneally infected with exponentially grown 
pneumococci $\left(5 \times 10^{3}\right.$ bacteria in $200 \mu$ I PBS supplemented with $1.0 \%$ heat inactivated FBS) as described [60].

\section{Co-infection mouse model}

In co-infection experiments $\sim 1-2 \times 10^{7} \mathrm{CFU}$ of bioluminescent D39/ux and D39/ux $\Delta p n r A$ were mixed at a 1:1 ratio and used as infection dosis to infect two groups of CD-1 mice $(\mathrm{n}=14)$ intranasally. At time points $24 \mathrm{~h}$ and $48 \mathrm{~h}$ post infection the bacterial load (CFU) of D39/ux and D39/ux $\Delta p n r A$ in the nasopharynx and bronchi was quantified as described previously [12]. Briefly, after re-isolation of the bacteria the output of wild-type D39/ux and mutant D39/ux $\Delta p n r A$ was determined on selective blood agar plates (Oxoid, Basingstoke, UK) containing kanamycin or erythromycin. The competitive index (Cl) was then calculated as the ratio of mutant to wild-type output CFU divided by the mutant to wild-type input CFU. A value of 1 indicates identical output CFU of wild-type and mutant bacteria, while $\mathrm{Cl}$ values lower than 1 indicated a higher output of wild-type bacteria as described [12].

\section{Ethics statement.}

All animal experiments were conducted in strict accordance with the recommendations in the Guide for the Care and Use of Laboratory Animals (National Research Council, USA), the guidelines of the ethics committee at The University of Greifswald and the German regulations of the Society for Laboratory Animal Science (GVSOLAS) and the European Health Law of the Federation of Laboratory Animal Science Associations (FELASA). All experiments were approved by the Landesamt für Landwirtschaft, 
Lebensmittelsicherheit und Fischerei Mecklenburg-Vorpommern (LALLFV M-V, Rostock, Germany) and the LALLFV M-V ethical board (LALLF M-V permit no. 7221.31.1-019/11 and 7221.3-1-056/16). All efforts were made to minimize suffering, ensure the highest ethical standard and also to adhere to the $3 \mathrm{R}$ principle (reduction, refinement and replacement).

\section{Acknowledgement}

This work was supported by grants from the Deutsche Forschungsgemeinschaft DFG GRK 1870 (to SH and ML), the Bundesministerium für Bildung und Forschung (BMBF) -

Zwanzig20 - InfectControl 2020 - project VacoME - FKZ 03ZZ0816A to SH, the Federal Excellence Initiative of Mecklenburg Western Pomerania and European Social Fund (ESF) Grant Kolnfekt (ESF_14-BM-A55-0001_16) to SH and by financial support from the Spanish Ministry of Science, Innovation and Universities BFU2017-90030-P to JAH. We thankfully acknowledge the technical support provided by staff of the beamline XALOC at the ALBA synchrotron facility (Barcelona, Spain) and Teresia Hallström (NanoTemper, Munich, Germany).

\section{Authors contributions}

S.H. and J.A.H designed the experiments; M.R.A, M.T.B, F.J., F.V., P.W., T.P.K., R.M., St.H., M.L. performed the research; S.H., J.A.H., M.R.A, M.T.B, and M.L. analyzed the data; S.H. and J.A.H. wrote the paper. All authors discussed the results, edited and approved the paper. 


\section{Declaration of competing interest}

The authors declare no financial conflict of interest

\section{Supplementary data}

Figure S1. Genomic organization of the pnr-operon and pnr gene expression analysis by qPCR.

Figure S2. Comparison of the open and closed conformations of Cluster B in Class I of the Substrate Binding Proteins.

Figure S3. 2Fo-Fc electron density maps for nucleosides in the PnrA:nucleoside complexes.

Figure S4. Initial Fo-Fc electron density maps for nucleosides in the PnrA:nucleoside complexes

Figure S5. Superimposition of the PnrA:nucleoside structures.

Figure S6. Stereo view showing interactions between PnrA and the five different nucleosides.

Figure S7. Comparison of nucleoside binding in PnrA and TmpC complexes

Figure S8. Fluorescence microscopy of phagocytosed pneumococci.

TABLE S1. Strains and plasmids list

TABLE S2. Primers used for qRT-PCR

TABLE S3. Crystallographic data.

TABLE S4. Distances of the $\mathrm{H}$ bonds in nucleoside recognition by PnrA 


\section{References}

[1] Kilstrup M, Hammer K, Ruhdal Jensen P, Martinussen J. Nucleotide metabolism and its control in lactic acid bacteria. FEMS microbiology reviews. 2005;29:555-90.

[2] Munch-Petersen A, Mygind B. Nucleoside transport systems in Escherichia coli K12: specificity and regulation. Journal of cellular physiology. 1976;89:551-9.

[3] Hantke K. Phage T6--colicin K receptor and nucleoside transport in Escherichia coli. FEBS letters. 1976;70:109-12.

[4] Saxild HH, Andersen LN, Hammer K. Dra-nupC-pdp operon of Bacillus subtilis: nucleotide sequence, induction by deoxyribonucleosides, and transcriptional regulation by the deoR-encoded DeoR repressor protein. Journal of bacteriology. 1996;178:424-34.

[5] Johansen LE, Nygaard P, Lassen C, Agerso Y, Saxild HH. Definition of a second Bacillus subtilis pur regulon comprising the pur and xpt-pbuX operons plus pbuG, nupG (yxjA), and pbuE (ydhL). Journal of bacteriology. 2003;185:5200-9.

[6] Feldman C, Anderson R. The Role of Streptococcus pneumoniae in Community-Acquired Pneumonia. Semin Respir Crit Care Med. 2016;37:806-18.

[7] Loughran AJ, Orihuela CJ, Tuomanen El. Streptococcus pneumoniae: Invasion and Inflammation. Microbiol Spectr. 2019;7.

[8] Musher DM, Thorner AR. Community-acquired pneumonia. N Engl J Med. 2014;371:1619-28.

[9] O'Brien KL, Wolfson LJ, Watt JP, Henkle E, Deloria-Knoll M, McCall N, et al. Burden of disease caused by Streptococcus pneumoniae in children younger than 5 years: global estimates. Lancet (London, England). 2009;374:893-902.

[10] Hoskins J, Alborn WE, Jr., Arnold J, Blaszczak LC, Burgett S, DeHoff BS, et al. Genome of the bacterium Streptococcus pneumoniae strain R6. Journal of bacteriology. 2001;183:5709-17.

[11] Kohler S, Voss F, Gomez Mejia A, Brown JS, Hammerschmidt S. Pneumococcal lipoproteins involved in bacterial fitness, virulence, and immune evasion. FEBS letters. 2016;590:3820-39.

[12] Schulz C, Gierok P, Petruschka L, Lalk M, Mader U, Hammerschmidt S. Regulation of the arginine deiminase system by ArgR2 interferes with arginine metabolism and fitness of Streptococcus pneumoniae. mBio. 2014;5.

[13] Hartel T, Klein M, Koedel U, Rohde M, Petruschka L, Hammerschmidt S. Impact of glutamine transporters on pneumococcal fitness under infection-related conditions. Infect Immun. 2011;79:4458.

[14] Schulz C, Hammerschmidt S. Exploitation of physiology and metabolomics to identify pneumococcal vaccine candidates. Expert Rev Vaccines. 2013;12:1061-75.

[15] Deka RK, Brautigam CA, Yang XF, Blevins JS, Machius M, Tomchick DR, et al. The PnrA (Tp0319; TmpC) lipoprotein represents a new family of bacterial purine nucleoside receptor encoded within an ATP-binding cassette (ABC)-like operon in Treponema pallidum. The Journal of biological chemistry. 2006;281:8072-81.

[16] Martinussen J, Sorensen C, Jendresen CB, Kilstrup M. Two nucleoside transporters in Lactococcus lactis with different substrate specificities. Microbiology (Reading, England). 2010;156:3148-57.

[17] Webb AJ, Hosie AH. A member of the second carbohydrate uptake subfamily of ATP-binding cassette transporters is responsible for ribonucleoside uptake in Streptococcus mutans. Journal of bacteriology. 2006;188:8005-12.

[18] Bidossi A, Mulas L, Decorosi F, Colomba L, Ricci S, Pozzi G, et al. A functional genomics approach to establish the complement of carbohydrate transporters in Streptococcus pneumoniae. PloS one. 2012;7:e33320.

[19] Schneider E. ABC transporters catalyzing carbohydrate uptake. Research in microbiology. 2001;152:303-10.

[20] Saxena S, Khan N, Dehinwal R, Kumar A, Sehgal D. Conserved surface accessible nucleoside ABC transporter component SP0845 is essential for pneumococcal virulence and confers protection in vivo. PloS one. 2015;10:e0118154.

[21] Voss F, Kohler TP, Meyer T, Abdullah MR, van Opzeeland FJ, Saleh M, et al. Intranasal Vaccination With Lipoproteins Confers Protection Against Pneumococcal Colonisation. Frontiers in immunology. 2018;9:2405. 
[22] Pribyl T, Moche M, Dreisbach A, Bijlsma JJ, Saleh M, Abdullah MR, et al. Influence of impaired lipoprotein biogenesis on surface and exoproteome of Streptococcus pneumoniae. Journal of proteome research. 2014;13:650-67.

[23] Brown JS, Ogunniyi AD, Woodrow MC, Holden DW, Paton JC. Immunization with components of two iron uptake $A B C$ transporters protects mice against systemic Streptococcus pneumoniae infection. Infect Immun. 2001;69:6702-6.

[24] Johnson SE, Dykes JK, Jue DL, Sampson JS, Carlone GM, Ades EW. Inhibition of pneumococcal carriage in mice by subcutaneous immunization with peptides from the common surface protein pneumococcal surface adhesin a. The Journal of infectious diseases. 2002;185:489-96.

[25] Whalan RH, Funnell SGP, Bowler LD, Hudson MJ, Robinson A, Dowson CG. PiuA and PiaA, iron uptake lipoproteins of Streptococcus pneumoniae, elicit serotype independent antibody responses following human pneumococcal septicaemia. FEMS Immunology \& Medical Microbiology. 2005;43:73-80.

[26] Garmory HS, Titball RW. ATP-Binding Cassette Transporters Are Targets for the Development of Antibacterial Vaccines and Therapies. Infection and Immunity. 2004;72:6757-63.

[27] Berry AM, Paton JC. Sequence heterogeneity of PsaA, a 37-kilodalton putative adhesin essential for virulence of Streptococcus pneumoniae. Infection and Immunity. 1996;64:5255-62.

[28] Ogunniyi AD, Mahdi LK, Trappetti C, Verhoeven N, Mermans D, Van der Hoek MB, et al. Identification of genes that contribute to the pathogenesis of invasive pneumococcal disease by in vivo transcriptomic analysis. Infect Immun. 2012;80:3268-78.

[29] Ogunniyi AD, Paton JC. Vaccine Potential of Pneumococcal Proteins. In: Brown J, Hammerschmidt $\mathrm{S}$, Orihuela C, editors. Streptococcus pneumoniae Molecular Mechanisms of Host-Pathogen Interactions. 1 ed. Amsterdam: Elsevier; 2015. p. 59-71.

[30] Song X-M, Connor W, Jalal S, Hokamp K, Potter AA. Microarray analysis of Streptococcus pneumoniae gene expression changes to human lung epithelial cells. Canadian Journal of Microbiology. 2008;54:189-200.

[31] Leonard A, Gierok P, Methling K, Gomez-Mejia A, Hammerschmidt S, Lalk M. Metabolic inventory of Streptococcus pneumoniae growing in a chemical defined environment. Int J Med Microbiol. 2018;308:705-12.

[32] Karlskas IL, Saleihan Z, Holo H, Mathiesen G, Eijsink VG. EF0176 and EF0177 from Enterococcus faecalis V583 are substrate-binding lipoproteins involved in ABC transporter mediated ribonucleoside uptake. Microbiology (Reading, England). 2015;161:754-64.

[33] Belitsky BR, Sonenshein AL. CodY-Mediated Regulation of Guanosine Uptake in Bacillus subtilis. Journal of bacteriology. 2011;193:6276-87.

[34] Martinussen J, Wadskov-Hansen SLL, Hammer K. Two Nucleoside Uptake Systems in Lactococcus lactis: Competition between Purine Nucleosides and Cytidine Allows for Modulation of Intracellular Nucleotide Pools. Journal of bacteriology. 2003;185:1503-8.

[35] Briles DE, Ades E, Paton JC, Sampson JS, Carlone GM, Huebner RC, et al. Intranasal immunization of mice with a mixture of the pneumococcal proteins PsaA and PspA is highly protective against nasopharyngeal carriage of Streptococcus pneumoniae. Infect Immun. 2000;68:796-800.

[36] Talkington DF, Brown BG, Tharpe JA, Koenig A, Russell H. Protection of mice against fatal pneumococcal challenge by immunization with pneumococcal surface adhesin A (PsaA). Microb Pathog. 1996;21:17-22.

[37] Yu J, Li B, Chen X, Lu J, Wang D, Gu T, et al. Comparison of Immunogenicity and Protection of Two Pneumococcal Protein Vaccines Based on PsaA and PspA. Infect Immun. 2018;86.

[38] Abdullah MR, Gutierrez-Fernandez J, Pribyl T, Gisch N, Saleh M, Rohde M, et al. Structure of the pneumococcal I,d-carboxypeptidase DacB and pathophysiological effects of disabled cell wall hydrolases DacA and DacB. Molecular microbiology. 2014;93:1183-206.

[39] Berntsson RP, Smits SH, Schmitt L, Slotboom DJ, Poolman B. A structural classification of substratebinding proteins. FEBS letters. 2010;584:2606-17.

[40] Mao B, Pear MR, McCammon JA, Quiocho FA. Hinge-bending in L-arabinose-binding protein. The "Venus's-flytrap" model. J Biol Chem. 1982;257:1131-3.

[41] Jeffrey GA. An Introduction to Hydrogen Bonding: Oxford University Press; 1997.

[42] Traut TW. Physiological concentrations of purines and pyrimidines. Mol Cell Biochem. 1994;140:122. 
[43] Ramakers BP, Pickkers P, Deussen A, Rongen GA, van den Broek P, van der Hoeven JG, et al. Measurement of the endogenous adenosine concentration in humans in vivo: methodological considerations. Curr Drug Metab. 2008;9:679-85.

[44] Chimalapati S, Cohen JM, Camberlein E, MacDonald N, Durmort C, Vernet T, et al. Effects of deletion of the Streptococcus pneumoniae lipoprotein diacylglyceryl transferase gene lgt on $A B C$ transporter function and on growth in vivo. PloS one. 2012;7:e41393.

[45] Petit CM, Brown JR, Ingraham K, Bryant AP, Holmes DJ. Lipid modification of prelipoproteins is dispensable for growth in vitro but essential for virulence in Streptococcus pneumoniae. FEMS microbiology letters. 2001;200:229-33.

[46] Hermans PW, Adrian PV, Albert C, Estevao S, Hoogenboezem T, Luijendijk IH, et al. The streptococcal lipoprotein rotamase $A(S \mid r A)$ is a functional peptidyl-prolyl isomerase involved in pneumococcal colonization. The Journal of biological chemistry. 2006;281:968-76.

[47] Cron LE, Bootsma HJ, Noske N, Burghout P, Hammerschmidt S, Hermans PW. Surface-associated lipoprotein PpmA of Streptococcus pneumoniae is involved in colonization in a strain-specific manner. Microbiology (Reading, England). 2009;155:2401-10.

[48] Leonard A, Lalk M. Infection and metabolism - Streptococcus pneumoniae metabolism facing the host environment. Cytokine. 2018;112:75-86.

[49] Gomez-Mejia A, Gamez G, Hirschmann S, Kluger V, Rath H, Bohm S, et al. Pneumococcal Metabolic Adaptation and Colonization Are Regulated by the Two-Component Regulatory System 08. mSphere. 2018;3.

[50] Pfaffl MW, Horgan GW, Dempfle L. Relative expression software tool (REST) for group-wise comparison and statistical analysis of relative expression results in real-time PCR. Nucleic acids research. 2002;30:e36.

[51] Dorries K, Lalk M. Metabolic footprint analysis uncovers strain specific overflow metabolism and Disoleucine production of Staphylococcus aureus COL and HG001. PloS one. 2013;8:e81500.

[52] Wider G, Dreier L. Measuring protein concentrations by NMR spectroscopy. J Am Chem Soc. 2006;128:2571-6.

[53] Kabsch W. Xds. Acta Crystallogr D Biol Crystallogr. 2010;66:125-32.

[54] Evans PR, Murshudov GN. How good are my data and what is the resolution? Acta Crystallogr D Biol Crystallogr. 2013;69:1204-14.

[55] McCoy AJ, Grosse-Kunstleve RW, Adams PD, Winn MD, Storoni LC, Read RJ. Phaser crystallographic software. J Appl Crystallogr. 2007;40:658-74.

[56] Emsley P, Lohkamp B, Scott WG, Cowtan K. Features and development of Coot. Acta Crystallogr D Biol Crystallogr. 2010;66:486-501.

[57] Afonine PV, Grosse-Kunstleve RW, Echols N, Headd JJ, Moriarty NW, Mustyakimov M, et al. Towards automated crystallographic structure refinement with phenix.refine. Acta Crystallogr D Biol Crystallogr. 2012;68:352-67.

[58] Jensch I, Gamez G, Rothe M, Ebert S, Fulde M, Somplatzki D, et al. PavB is a surface-exposed adhesin of Streptococcus pneumoniae contributing to nasopharyngeal colonization and airways infections. Molecular microbiology. 2010;77:22-43.

[59] Saleh M, Abdullah MR, Schulz C, Kohler T, Pribyl T, Jensch I, et al. Following in real time the impact of pneumococcal virulence factors in an acute mouse pneumonia model using bioluminescent bacteria. Journal of visualized experiments : JoVE. 2014:e51174.

[60] Saleh M, Bartual SG, Abdullah MR, Jensch I, Asmat TM, Petruschka L, et al. Molecular architecture of Streptococcus pneumoniae surface thioredoxin-fold lipoproteins crucial for extracellular oxidative stress resistance and maintenance of virulence. EMBO molecular medicine. 2013;5:1852-70.

[61] Vaguine AA, Richelle J, Wodak SJ. SFCHECK: a unified set of procedures for evaluating the quality of macromolecular structure-factor data and their agreement with the atomic model. Acta Crystallogr D Biol Crystallogr. 1999;55:191-205. 


\section{Figure Legends}

Figure 1. Growth behavior of $S$. pneumoniae in RPMImodi in presence and absence of nucleosides supplementation and surface abundance of PnrA (A) Immunoblot confirms the absence of PnrA in the isogenic PnrA-deficient pneumococcal strains when using specific anti-PnrA antibodies as primary antibody. The enolase was used as loading control in bacterial lysates and rPnrA (His6-tagged PnrA) as a further control protein.

(B and C) Surface abundance of PnrA and PsaA as assessed by flow cytometry. Antigen specific antibody binding was detected with goat-anti-mouse Alexa Fluor ${ }^{\circledR} 488$ coupled secondary antibodies. The mean fluorescence intensity (MFI) multiplied with the percentage of positive gated events is depicted in (B), while representative histograms are shown in (C). The mean values of at least three independent experiments are shown with error bars corresponding to SEM. AB: antibody; WT: wildtype.

(D) S. pneumoniae wild-type strains D39 and the isogenic $\Delta p n r A$ mutant were grown in RPMImodi without (left panel) and with a nucleosides mixture (right panel). Optical density $\left(\mathrm{OD}_{600 \mathrm{~nm}}\right)$ was measured every hour for 9 hours. The resulting growth curves show no significant difference in growth between the individual wild-type and its pnrAmutant in both media. 
Figure 2. ${ }^{1} \mathrm{H}-\mathrm{NMR}$ based quantification of nucleosides in pneumococcal cultures. The initial concentrations $(\mathrm{mmol} / \mathrm{l})$ of nucleosides provided in the cultured are indicated with a dashed line. During growth $\left(T_{1}=5 \mathrm{hrs}\right.$ and $\left.T_{2}=8 \mathrm{hrs}\right)(\mathrm{A})$ guanosine, $(\mathrm{B})$ adenosine and $(C)$ cytidine were found to be taken up by pneumococcal strains D39, D39 $\Delta c p s$ and the corresponding $\Delta p n r A$ deletion mutants. Uptake was experimentally measured by ${ }^{1} \mathrm{H}-\mathrm{NMR}$ analysis and could not be demonstrated for uridine and thymidine in (D) and (E). Experiments were carried out in independent biological triplicates $(n=3)$.

Figure 3. Nucleoside binding to PnrA.

The binding affinities of nucleosides to PnrA were determined by MST experiments. The lipoprotein and L,D-carboxypeptidase DacB [38] was used as a negative control (A-E). The concentration of NHS-RED -labeled PnrA and DacB was kept constant (20 nM), while the concentration of the non-labeled nucleosides ranged between $100 \mu \mathrm{M}-$ $0.0122 \mu \mathrm{M}$. The samples were loaded into Monolith NT.115 Premium Capillaries (NanoTemper Technologies) and the MST measurements were performed using the Monolith NT.115 (NanoTemper Technologies) at 80\% LED power and medium MST power. An MST-on time of $15 \mathrm{~s}$ was used for analysis, and a $\mathrm{K}_{d}$ was calculated from three independent measurements, error bars represent the standard deviation.

Figure 4. Three-dimensional structures of PnrA:nucleoside complexes.

(A) Cartoon showing the three-dimensional structure of PnrA: adenosine complex with each domain colored differently ( $\mathrm{N}$ domain in blue, and $\mathrm{C}$ domain in orange). Adenosine substrate is shown as green spheres. The three hinge regions (CR1-CR3) are labeled. 
Residues interacting with the nucleoside for the (B) PnrA: adenosine (complex 1), (C) PnrA: guanosine (complex 2) (D) PnrA: cytidine (complex 3), (E) PnrA: uridine (complex 4), and (F) PnrA: thymidine (complex 5). All the ligands are shown as capped sticks (C atoms in green) and labeled. Important residues in substrate recognition are drawn as capped sticks and labeled. Water molecules are drawn as red spheres. Polar interactions are represented as dashed lines.

Figure 5. $\mathrm{H}$-bond interactions and their classification as observed in the PnrA:nucleoside complexes. Critical residues, and atoms, involved in substrate recognition are shown for each PnrA complex. According to Jeffrey [41] H-bonds are classified as strong (distances from $2.20 \AA$ to $2.50 \AA$ ) colored in red, moderate (distances from $2.51 \AA$ to $3.20 \AA$ ) colored in orange and weak (distances from $3.21 \AA$ to $4.00 \AA$ ) colored in yellow. $\mathrm{H}$-bond distance was calculated as the average distance in the four independent monomers observed per complex. Expected maximal error in coordinates for each crystallographic complex was of $0.1 \AA$ for complex 3 (cytidine) and of $0.2 \AA$ for the rest of complexes (complexes $\mathbf{1 , 2 , 4}$ and $\mathbf{5}$ ) as calculated by SFCHECK [61] (see methods).

Figure 6. Effect of PnrA deficiency on uptake of S. pneumoniae D39 by professional phagocytes

J774 murine macrophages were infected for 30 min with non-encapsulated D39 or the isogenic pnrA-mutant with a multiplicity of infection (MOI) of 50 bacteria per macrophage. The recovery of intracellular pneumococcal survivors was quantified by 
applying the antibiotic protection assay and plating the bacteria on blood agar plates immediately after antibiotics treatment or 1, 2 or 3 hours later. Experiments were done four times and data represent the mean \pm SEM. (A) Absolute numbers of recovered bacteria and $(B)$ the number of bacteria at time point to was set to $100 \%$ for each strain to determine the survival rate inside the macrophages. ${ }^{*} P<0.05$; ${ }^{* *} P<0.01$, n.s. nonsignificant.

(C) Quantification of pneumococci that are extracellularly attached to or located intracellularly in macrophages.

Representative double immunofluorescence pictures (Scale bars $10 \mu \mathrm{m}$ ) are depicted in (D) and Fig. S7, where pneumococci were stained through incubation with polyclonal rabbit anti-pneumococci IgG followed by either labeling with goat anti-rabbit IgG coupled Alexa-Fluor-488 (green, extracellular) or goat anti-rabbit IgG coupled Alexa-Fluor-568 (red, intracellular) antibody. Time point $0 \mathrm{~h}$ represents the time point, when phagocytes have been incubated for 30 min with pneumococci, while at time point $1 \mathrm{~h}$ phagocytes were washed twice with infection medium and incubated further for $1 \mathrm{~h}$.

Figure 7. Impact of PnrA on pneumococcal colonization and lung infection (A) Kaplan-Meier survival curves of CD-1 mice intranasally infected with $S$. pneumoniae. Groups of mice $(n=10)$ were infected intranasally with $5 \times 10^{7} \mathrm{CFU}$ of S. pneumoniae D39/ux or D39/ux $\Delta$ pnrA. (B) Survival curves of CD-1 mice infected intraperitoneally with $5 \times 10^{3} \mathrm{CFU}$ of the S. pneumoniae strains D39/ux and D39/ux $\Delta p n r A$ (C) Spread of pneumococci in CD-1 mice after intranasal infection monitored by bioluminescence measurement. The distribution of bioluminescent pneumococci was 
visualized and measured at indicated time points and the luminescence intensity (photons/second) was determined using the IVIS ${ }^{\circledR}$ Spectrum system.

(D) CD-1 mice $(n=14)$ were co-infected with a mixture containing equal colony forming units (CFU of 1-2 x 107) of bioluminescent D39/ux and D39/ux $\Delta p n r A$. Twenty-four hours and $48 \mathrm{~h}$ post-infection mice were sacrificed and the CFU in the nasopharynx (NP: nasopharyngeal lavage), airways (BAL: bronchoalveolar lavage) and blood were counted after plating the bacteria on selective blood agar plates. A competitive index (Cl) values lower than 1 indicated that the wild-type outcompeted the pnrA-mutant. 
FIGURES

FIGURE 1

A
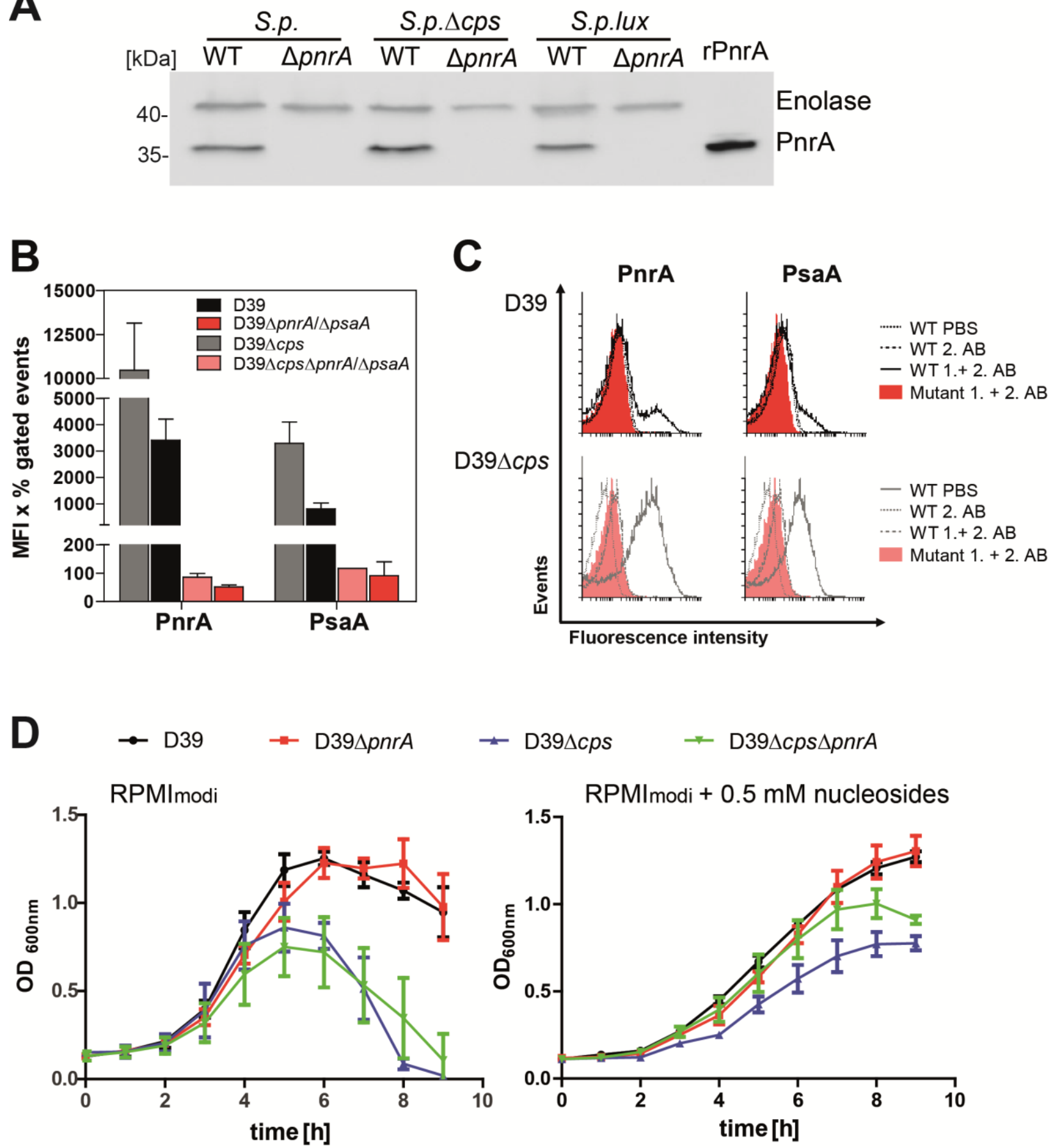

D39 4 cps

- D39 $c c p s \Delta p n r A$

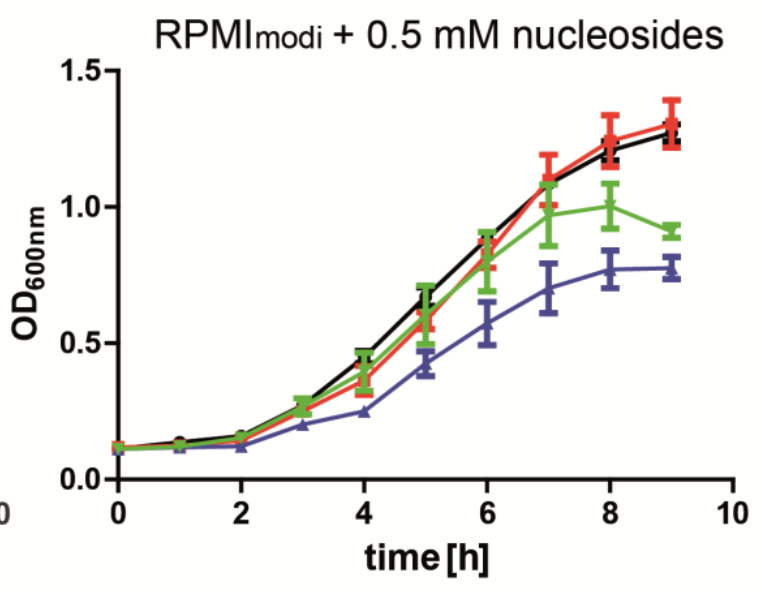


FIGURE 2
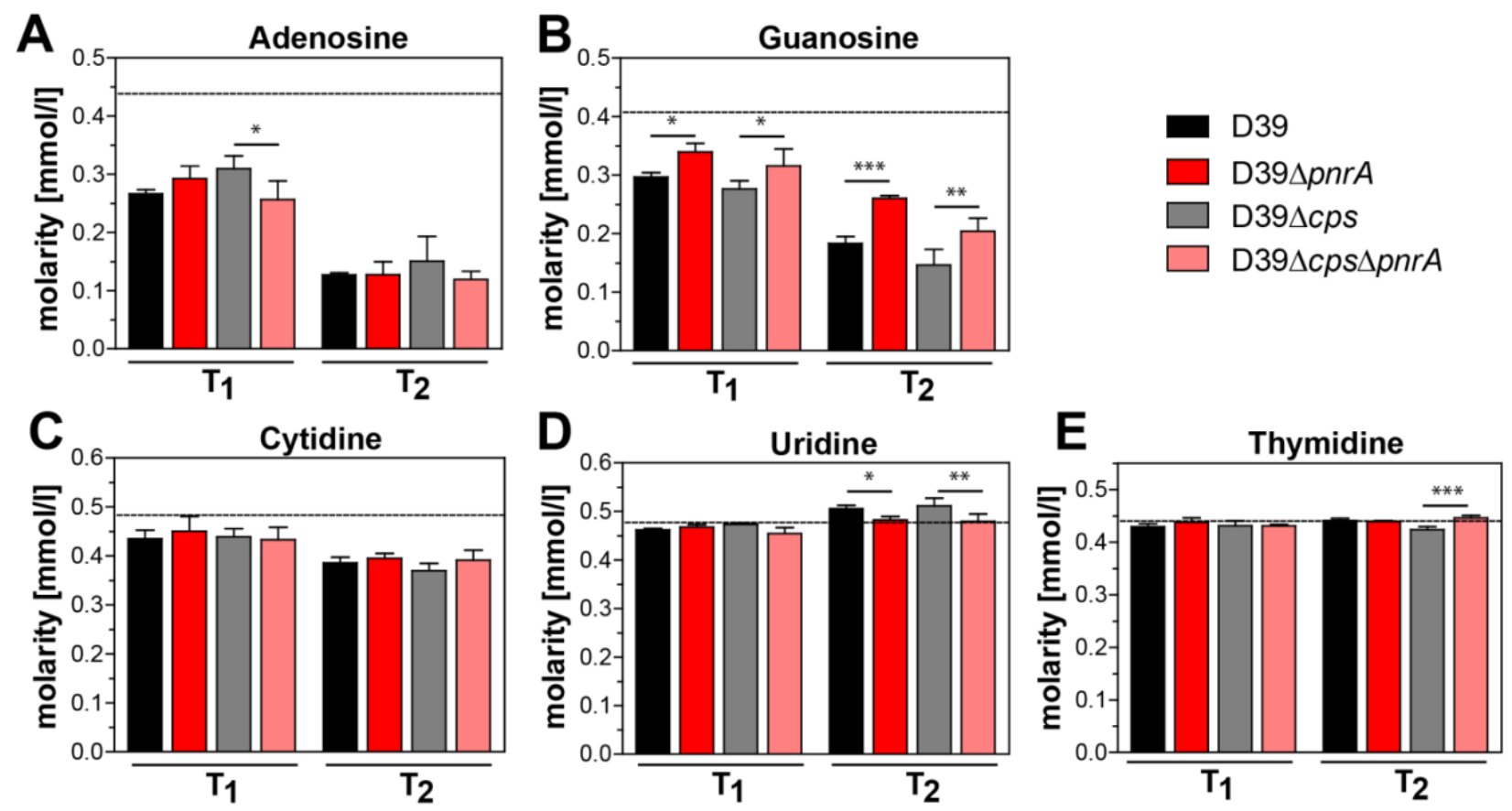
FIGURE 3
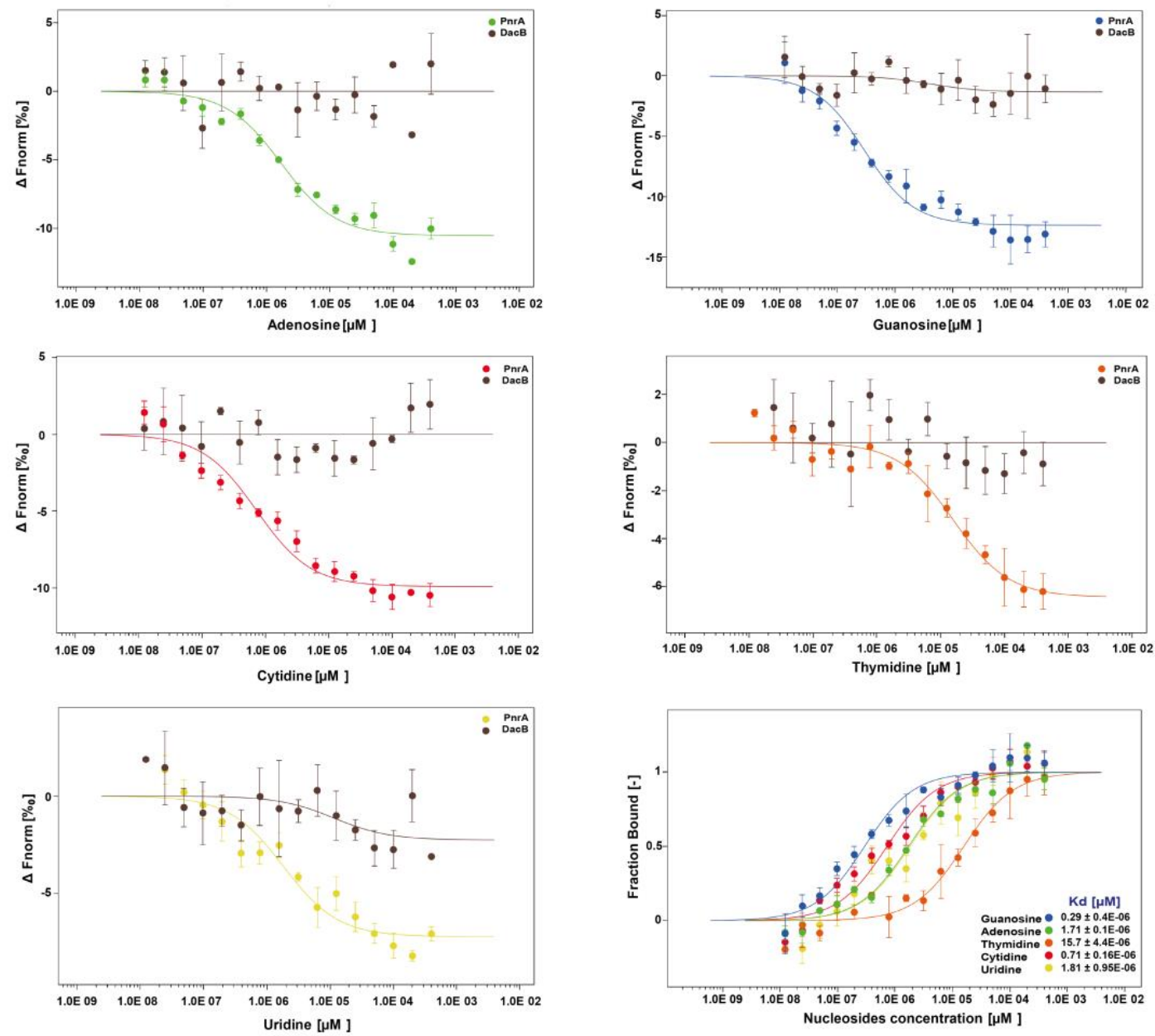
FIGURE 4

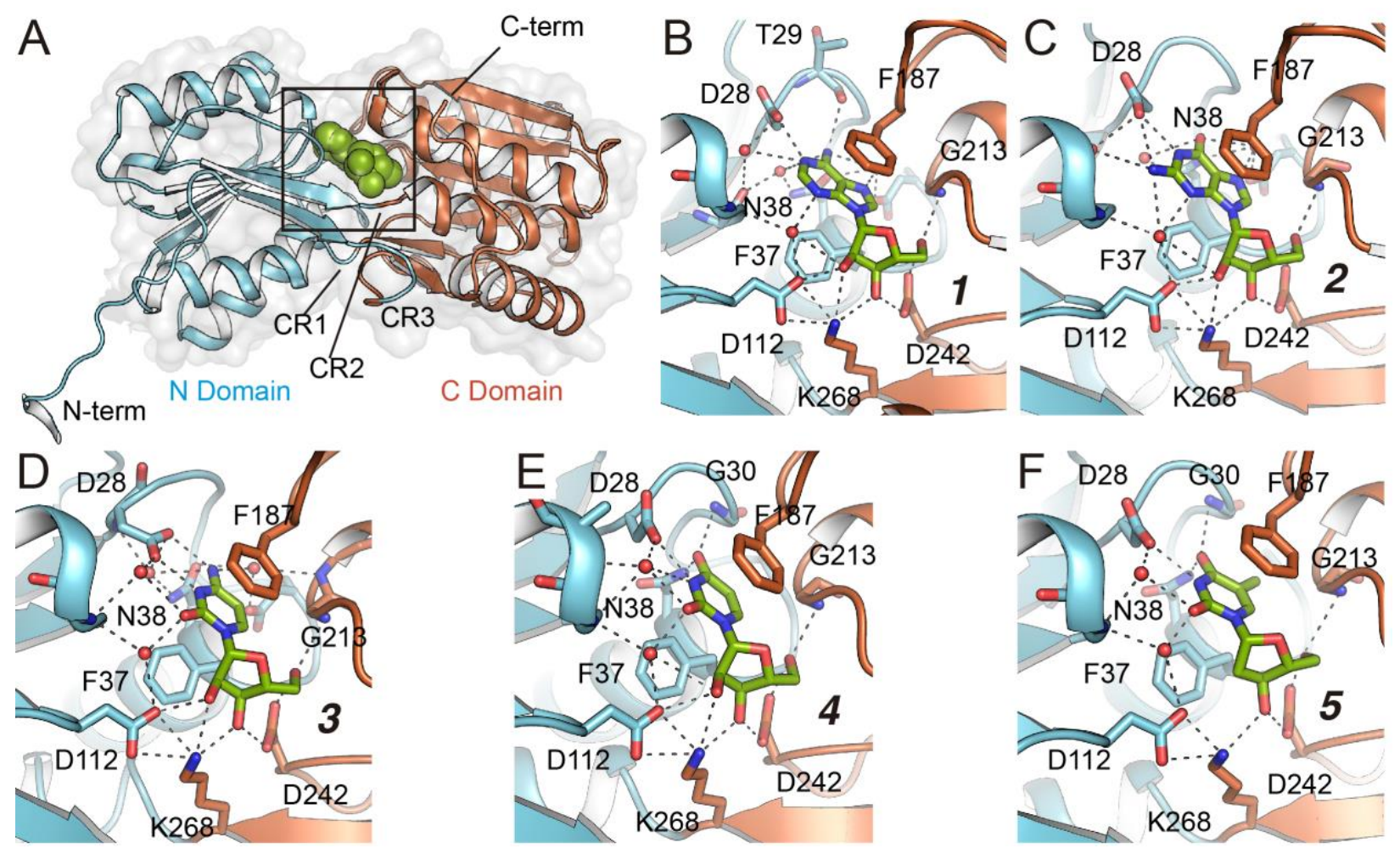


FIGURE 5

\begin{tabular}{|c|c|c|c|c|c|c|c|c|c|c|c|c|c|}
\hline \multirow{3}{*}{ PnrA } & \multicolumn{7}{|c|}{ purine/pyrimidine recognition } & \multicolumn{6}{|c|}{ ribose/deoxyribose recognition } \\
\hline & \multicolumn{3}{|c|}{ D28 } & \multirow{2}{*}{\begin{tabular}{|c|} 
T29 \\
0
\end{tabular}} & \multirow{2}{*}{$\frac{\text { G30 }}{N}$} & \multicolumn{2}{|c|}{ N38 } & \multirow{2}{*}{\begin{tabular}{|l|} 
D112 \\
O8
\end{tabular}} & \multicolumn{2}{|c|}{ K268 } & \multicolumn{2}{|c|}{ D242 } & \multirow{2}{*}{$\frac{G 213}{N}$} \\
\hline & O\&2 & $\mathrm{O} 82$ & Os1 & & & O 1 & N82 & & NS & NS & O\&1 & O82 & \\
\hline adenosine (1) & - & N1 & - & N6 & - & N6 & - & $\mathrm{O} 2^{\prime}$ & O2' & O3' & O3' & O5' & O5' \\
\hline guanosine (2) & N2 & N1 & 一 & - & O6 & - & O6 & O2' & O2' & O3' & O3' & O5' & O5' \\
\hline
\end{tabular}

\begin{tabular}{|c|c|c|c|c|c|c|c|c|c|c|c|c|c|}
\hline cytidine (3) & - & N3 & N4 & N2 & - & N2 & - & O2' & O2' & O3' & O3' & O5' & O5' \\
\hline uridine (4) & - & N3 & 一 & 一 & $\mathrm{O} 2$ & - & O4 & O2' & O2' & O3' & O3' & O5' & O5' \\
\hline thymidine (5) & - & N3 & 一 & - & $\mathrm{O} 2$ & - & $\mathrm{O} 4$ & - & - & O3' & O3' & O5' & O5' \\
\hline
\end{tabular}

Strong (2.20-2.50 ^)

Moderate (2.51-3.20 ̊̊)

Weak (3.21-4.00 $)$ 
FIGURE 6
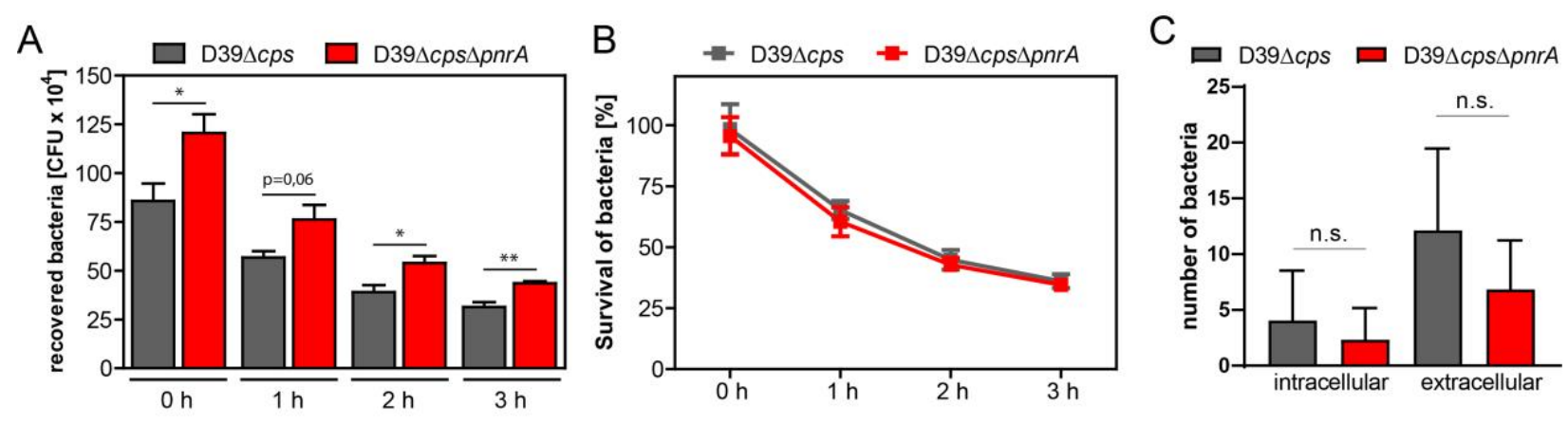

D

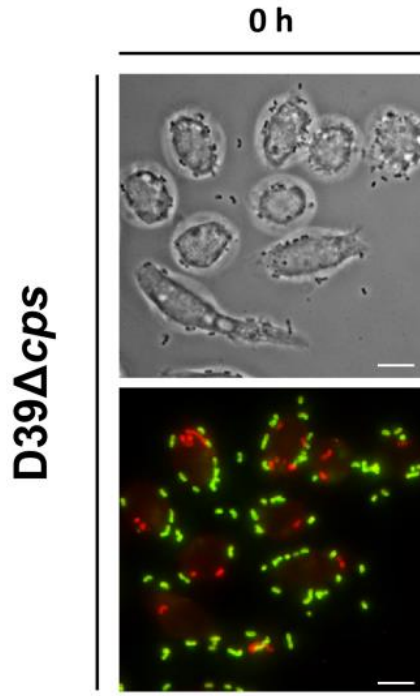

$1 \mathrm{~h}$

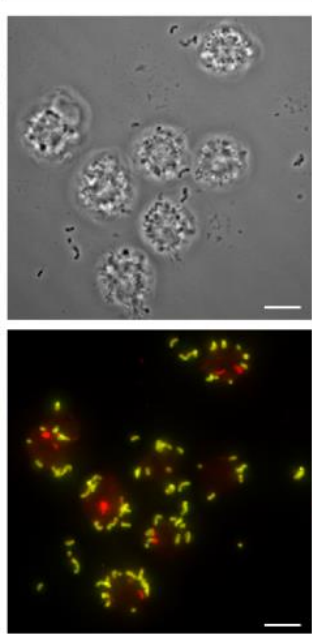

$2 \mathrm{~h}$

$3 \mathrm{~h}$
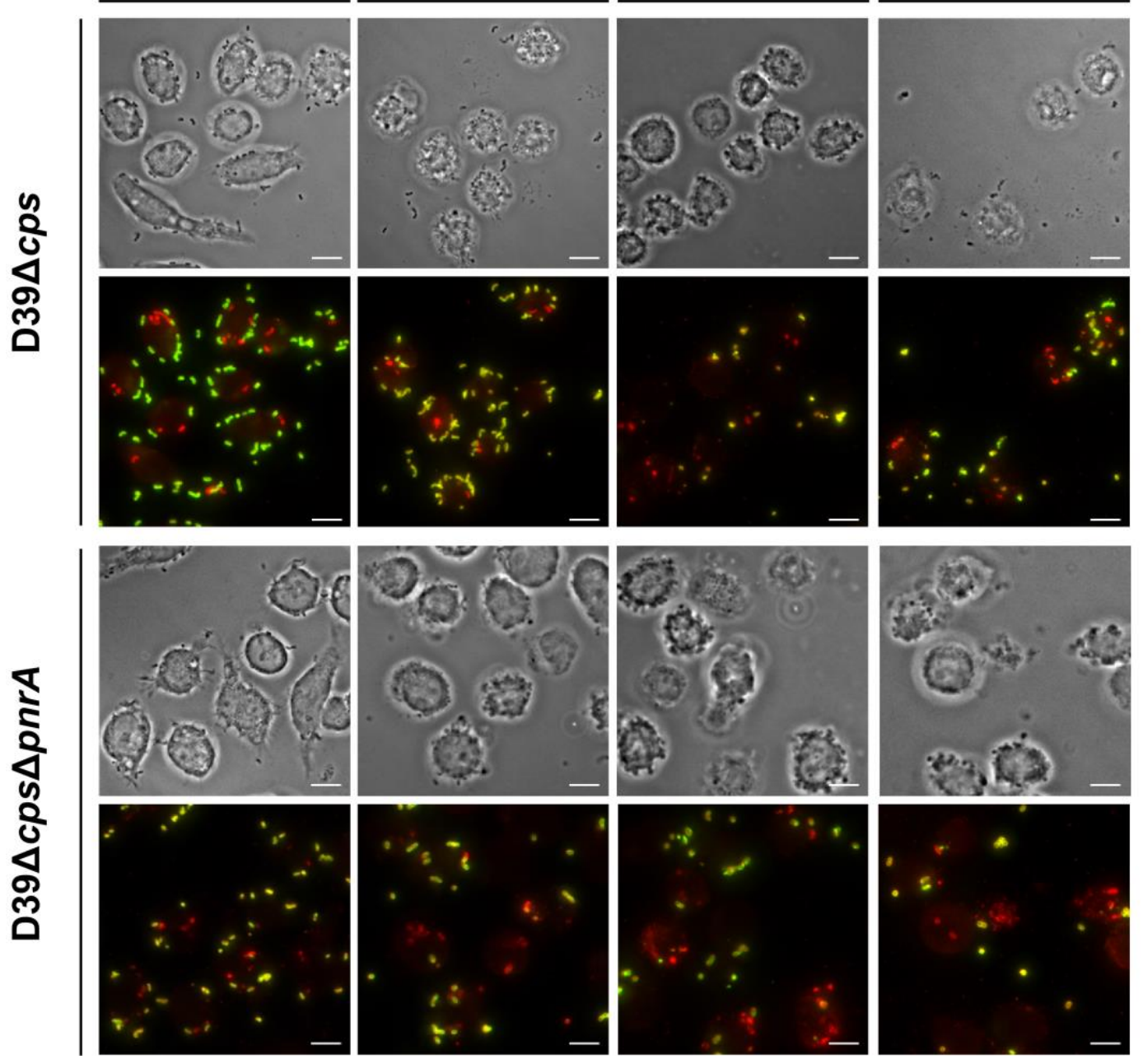
FIGURE 7

A

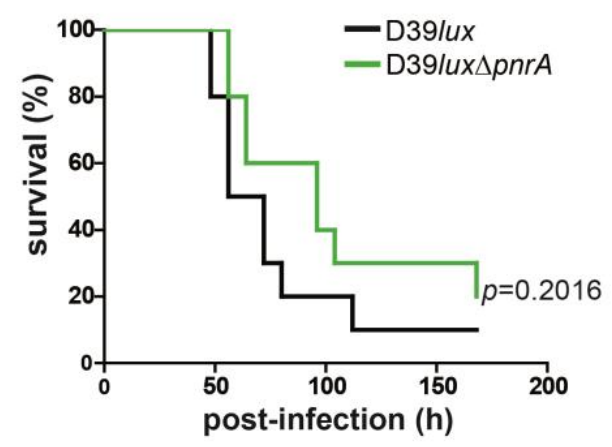

C
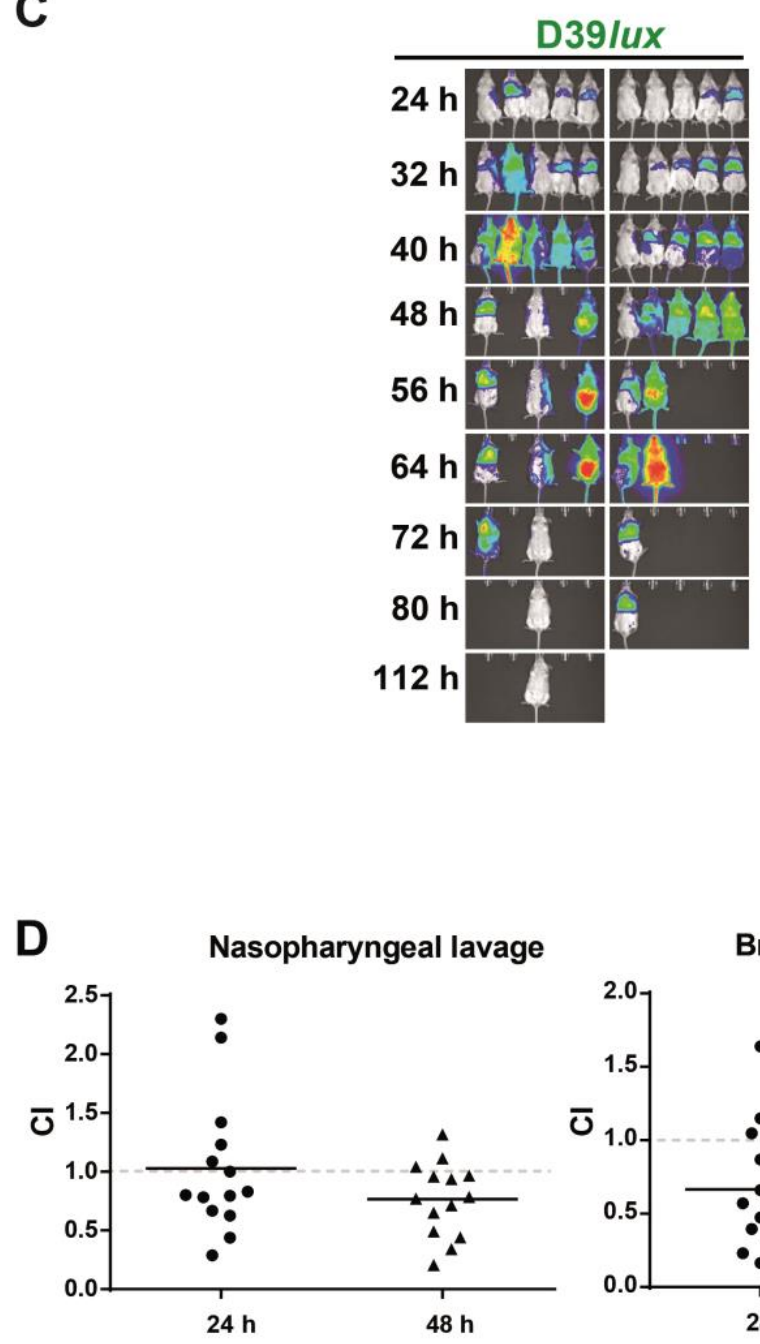

B
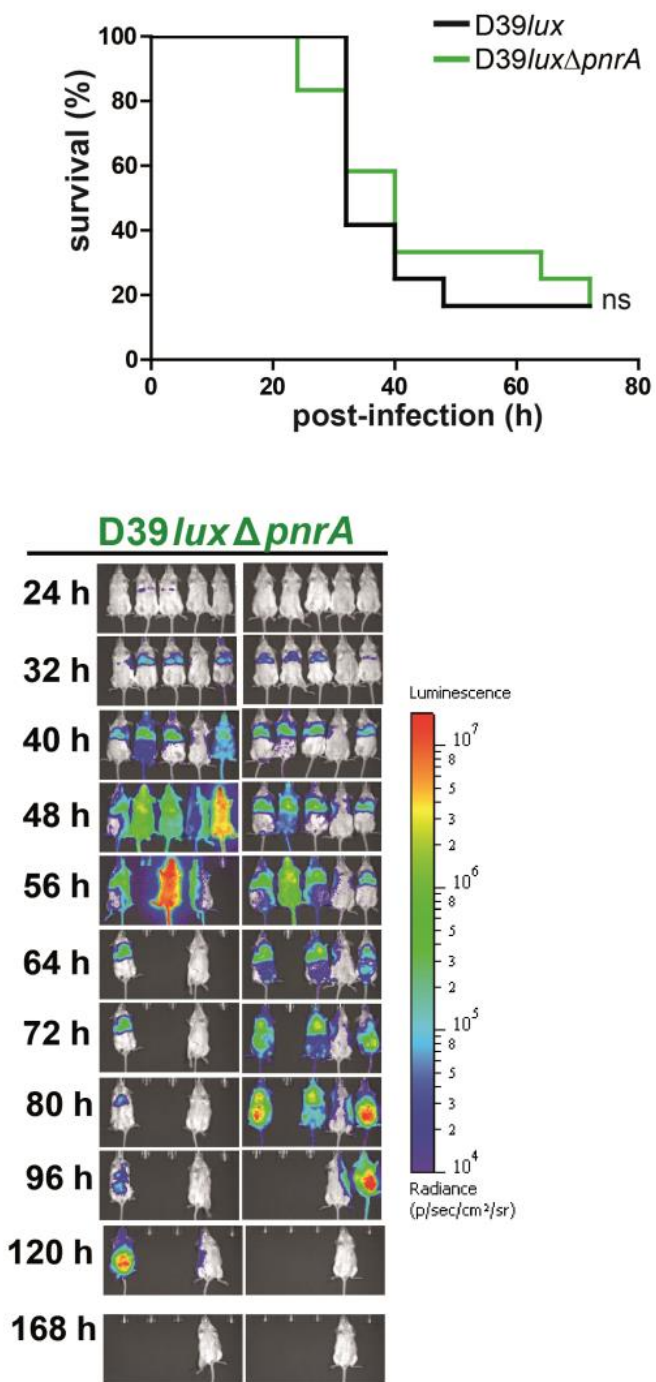